OPEN ACCESS

Edited by: Milena Dropa

University of São Paulo, Brazi

Reviewed by:

Luigi Principe

Azienda Sanitaria Provinciale di

Crotone, Italy

Jaroslav Hrabak,

Charles University, Czechia

*Correspondence:

Anand Anbarasu

aanand@vit.ac.in

Balaji Veeraraghavan

vbalaji@cmcvellore.ac.in

${ }^{\dagger}$ These authors have contributed equally to this work

Specialty section: This article was submitted to

Clinical Microbiology,

a section of the journal

Frontiers in Cellular and

Infection Microbiology

Received: 14 May 2021

Accepted: 04 August 2021

Published: 13 September 2021

Citation:

Shankar C, Basu S, Lal B,

Shanmugam S, Vasudevan $K$,

Mathur P, Ramaiah S, Anbarasu A and Veeraraghavan $B$ (2021) Aerobactin

Seems To Be a Promising Marker

Compared With Unstable RmpA2 for the Identification of Hypervirulent

Carbapenem-Resistant Klebsiella

pneumoniae: In Silico and

In Vitro Evidence.

Front. Cell. Infect. Microbiol. 11:709681.

doi: 10.3389/fcimb.2021.709681

\section{Aerobactin Seems To Be a Promising Marker Compared With Unstable RmpA2 for the Identification of Hypervirulent Carbapenem-Resistant Klebsiella pneumoniae: In Silico and In Vitro Evidence}

\author{
Chaitra Shankar ${ }^{1 \dagger}$, Soumya Basu ${ }^{2 \dagger}$, Binesh Lal ${ }^{1}$, Sathiya Shanmugam ${ }^{1}$, \\ Karthick Vasudevan ${ }^{1}$, Purva Mathur ${ }^{3}$, Sudha Ramaiah ${ }^{2}$, Anand Anbarasu ${ }^{2 *}$ \\ and Balaji Veeraraghavan ${ }^{1 *}$ \\ ${ }^{1}$ Department of Clinical Microbiology, Christian Medical College and Hospital, Vellore, India, ${ }^{2}$ Medical \& Biological \\ Computing Laboratory, School of Biosciences \& Technology, Vellore Institute of Technology, Vellore, India, ${ }^{3}$ Department of \\ Laboratory Medicine, Jai Prakash Narayan Apex, Trauma Centre, All India Institute of Medical Sciences, New Delhi, India
}

Background: The incidence of hypervirulent (hv) carbapenem-resistant (CR) Klebsiella pneumoniae (Kp) is increasing globally among various clones and is also responsible for nosocomial infections. The CR-hvKp is formed by the uptake of a virulence plasmid by endemic high-risk clones or by the uptake of plasmids carrying antimicrobial resistance genes by the virulent clones. Here, we describe CR-hvKp from India belonging to high-risk clones that have acquired a virulence plasmid and are phenotypically unidentified due to lack of hypermucoviscosity.

Methods: Twenty-seven CRKp isolates were identified to possess rmpA2 by wholegenome sequencing; and resistance and virulence determinants were characterized. By in silico protein modeling (and validation), protein backbone stability analysis, and coarse dynamics study, the fitness of RmpA, RmpA2, and aerobactin-associated proteins-lucA and lutA, were determined to establish a reliable marker for clinical identification of CR-hvKp.

Results: The CR-hvKp belonged to multidrug-resistant (MDR) high-risk clones such as CG11, CG43, ST15, and ST231 and carried OXA-232 as the predominant carbapenemase followed by NDM. The virulence plasmid belonged to IncHI1B replicon type and carried frameshifted and truncated $r m p A$ and $r m p A 2$. This resulted in a lack of hypermucoviscous phenotype. However, functional aerobactin was expressed in all highrisk clones. In silico analysis portrayed that lucA and lutA were more stable than classical RmpA. Furthermore, lucA and lutA had lower conformational fluctuations in the functional domains than the non-functional RmpA2, which increases the fitness cost of the latter for its maintenance and expression among CR-hvKp. Hence, RmpA and RmpA2 are likely to be lost among CR-hvKp owing to the increased fitness cost while coding for essential antimicrobial resistance and virulence factors. 
Conclusion: Increasing incidence of convergence of AMR and virulence is observed among $K$. pneumoniae globally, which warrants the need for reliable markers for identifying CR-hvKp. The presence of non-functional RmpA2 among high-risk clones highlights the significance of molecular identification of CR-hvKp. The negative string test due to non-functional RmpA2 among CR-hvKp isolates challenges phenotypic screening and faster identification of this pathotype. This can potentially be counteracted by projecting aerobactin as a stable, constitutively expressed, and functional marker for rapidly evolving CR-hvKp.

Keywords: Klebsiella pneumoniae, hypervirulent, aerobactin, rmpA2, carbapenem resistance, OXA-232, structure, stability

\section{INTRODUCTION}

Hypervirulent (Hv) Klebsiella pneumoniae (Kp) is a notorious pathogen causing a wide spectrum of infections in immunocompetent patients as well as immunosuppressed patients (Shon et al., 2013). This pathotype is being increasingly reported in the last decade among communityand healthcare-associated infections and has acquired antimicrobial resistance, unlike its former counterpart (Zhan et al., 2017; Hao et al., 2020; Li et al., 2021). The virulence plasmid described earlier such as the pLVPK and pK2044 lacked genes coding for antimicrobial resistance and typically belonged to IncHI1B (Chen et al., 2004). However, recent reports suggest the acquisition of virulence plasmids by multidrug-resistant (MDR) $\mathrm{Kp}$ and hence insertion of antimicrobial resistance genes (ARGs) such as $b l a_{\mathrm{KPC}-2}$ and catAl into the virulence plasmid (Dong et al., 2018; Chen et al., 2020; Shankar et al., 2020a). In addition, reports of fusion/mosaic plasmids that carry two replicons such as IncHI1B-IncFIB and IncFIIK-IncFIBK are emerging, which code for antimicrobial resistance determinants as well as virulence genes (Lam et al., 2019; Turton et al., 2019). Furthermore, HvKp is no longer confined to selected clones but is reported among regional endemic clones such as ST11 in China and ST147 in Europe (Turton et al., 2019; Li et al., 2021). Hence, the threat of these superbugs is increasing, and this pathotype is evolving at a tremendous pace.

Traditionally, string test, a phenotypic screening marker, was used to identify hvKp, but recent studies have revealed poor sensitivity and specificity in identifying hvKp. Therefore, string test is no longer valuable in the identification of hvKp (Tan et al., 2014). Amidst the various genomic modifications that the hvKp has undergone over the last decade, the markers for genomic identification have remained a constant despite a lack of clear definition. A combination of genes $r m p A, r m p A 2$, iucA, iroB, and iroN (Russo and MacDonald, 2020; Hu et al., 2021; Russo et al., 2021) has been used in identifying hvKp along with determining the pathogenicity in virulence models using mice or Galleria mellonella.

In India, high rates of MDR and extensively drug-resistant (XDR) $\mathrm{Kp}$ are frequent causes of nosocomial infections, and they often carry NDM and OXA48-like carbapenemases alone or in combination (Veeraraghavan et al., 2017; Shankar et al., 2021).
The common clones of carbapenem-resistant Kp (CRKp) in India include ST231, the endemic clone, and other international highrisk clones such as ST11, ST14, ST15, and ST147 (Shankar et al., 2021). Conventionally, these CRKp do not carry the virulence plasmid; instead, they are characterized by the presence of four to five plasmids that carry ARGs. With advancement of genomics in bacteriology, the convergence of CRKp and HvKp to form CRHvKp has been identified in various countries including India (Lam et al., 2019; Turton et al., 2019; Tang et al., 2020). In the present study, we report CRKp belonging to high-risk international clones in India that acquired the virulence plasmid-carrying frameshifted rmpA2.

Although previous studies have reported the correlation of rmpA and rmpA2 as markers for hypervirulent and hypermucoviscous pathotypes, there is a dearth of comprehensive correlations between the constitutive virulence markers for hvKp, viz., $r m p A, r m p A 2$, and aerobactin, based on "genome-structure" perspectives. Our research group has extensively worked on genomics (Jacob et al., 2019; Shankar et al., 2020a; Vasudevan et al., 2020; Shankar et al., 2021) and in silico structural analysis (Lavanya et al., 2013; Lavanya et al., 2014; Lavanya et al., 2015) to decipher the functional circuitry of various pathogenic proteins. The present study adopted a combination of whole-genome sequencing (WGS), genomic analysis, computational modeling, and structural dynamics studies, which have not been used previously to assess the concerned virulence markers as per our knowledge. We aimed to understand the impact of rmpA2 mutations on the fitness among CR-hvKp based on the comparative structure-function profiles of RmpA, RmpA2, and aerobactin (IucA and IutA). This study will potentially lead to identifying a sustainable molecular marker for CR-hvKp in the context of deleterious mutations and molecular fitness, which can subsequently be used for clinical identification of CR-hvKp strains.

\section{MATERIALS AND METHODS}

\section{In-Vitro Methods}

\section{Bacterial Isolates and Clinical Details of Patients}

Twenty-seven K. pneumoniae identified during 2017-2019 at the Department of Clinical Microbiology, Christian Medical College and Hospital, Vellore, and the Department of Laboratory 
Medicine, Jai Prakash Narayan Apex, Trauma Centre, AIIMS, New Delhi, were included in the study. The isolates were obtained from clinical specimens such as blood $(\mathrm{n}=13)$, cerebrospinal fluid $(\mathrm{n}=4)$, broncho-alveolar lavage $(\mathrm{n}=8)$, and pus $(n=2)$. String test was performed to screen for the hypermucoviscous phenotype followed by screening of $r m p A$ and $r m p A 2$ by PCR to determine the hypervirulent strains (Brisse et al., 2009). Antimicrobial susceptibility testing against various first and second-line antimicrobials such as piperacillin/ tazobactam, ceftazidime, cefepime, meropenem, imipenem, gentamicin, amikacin, ciprofloxacin, and minocycline was performed by Kirby Bauer disc diffusion and interpreted according to Clinical and Laboratory Standards Institute (CLSI) guidelines (CLSI 2017-2019). The isolates were defined as MDR if they were resistant to more than one agent in at least three classes of antimicrobials (Magiorakos et al., 2012). Colistin susceptibility was determined using broth microdilution to obtain the minimum inhibitory concentrations (MICs) and interpreted according to European Committee on Antimicrobial Susceptibility Testing (EUCAST) guidelines. In vitro fitness of the study isolates was assessed using growth curve assay as previously described (Bachman et al., 2015).

Clinical details of the patients were obtained from electronic medical records prospectively, and hence, informed consent could not be obtained. The patient outcomes, therapeutic regimen, duration of hospital stay, comorbidities, and nature of infection were analyzed. Hospital-acquired infection (HAI) was defined as a positive culture with $K$. pneumoniae after $48 \mathrm{~h}$ of admission to hospital. Community-acquired infection (CAI) was defined as a positive culture with $K$. pneumoniae before $48 \mathrm{~h}$ on admission to hospital (Shankar et al., 2018).

\section{DNA Extraction and Genome Sequencing}

Total genomic DNA was extracted from the pelleted cells using Wizard DNA purification kit (Promega, WI, USA) as per the manufacturer's protocol. Extracted DNA was quantified using NanoDrop One spectrophotometry (Thermo Fisher Scientific, MA, USA) and Qubit 3.0 fluorometry (Life Technologies, CA, USA) and stored at $-20^{\circ} \mathrm{C}$ until further use.

Sequencing library was prepared using the Nextra DNA Flex library preparation kit (Illumina, San Diego, CA) as per the manufacturer's instructions. Subsequently, the paired-end library was subjected to sequencing on a HiSeq 2500 platform (Illumina, USA) generating $2 \times 150$-bp reads. Sequencing reads with a PHRED quality score below 20 were discarded; and adapters were trimmed using cutadapt v1.8.1 and assessed with FastQC v0.11.4.

Long-read sequencing was carried out using Oxford NanoporeMinION platform with FLO-MIN106 R9 flow cell (Oxford Nanopore Technologies, Oxford, UK). Long-read DNA library was prepared using the SQK-LSK108 ligation sequencing kit (v.R9) along with ONT EXP-NBD103 Native Barcode Expansion kit following the manufacturer's protocol (Oxford Nanopore Technologies, Oxford, UK). The library was loaded onto the flow cells and run for $48 \mathrm{~h}$ using the standard MinKNOW software. The Fast5 files generated from MinION sequencing were subjected to base calling using Guppy (https:// github.com/gnetsanet/ONT-GUPPY; accessed in January 2020).

\section{Genome Assembly and Evaluation}

Draft genome sequence data generated using Illumina were assembled using SPAdes (v.3.13.0) (Bankevich et al., 2012). Complete and highly accurate assembly was achieved using hybrid de novo assembly approach (Vasudevan et al., 2020). The nanopore long reads were error-corrected with the standalone Canu error correction tool (v.1.7) and assembled using the Unicycler hybrid assembly pipeline (v 0.4.6) with the default settings (Koren et al., 2017; Wick et al., 2017). The obtained genome sequence was polished using high-quality Illumina reads as described previously (Walker et al., 2014). The assembled complete genome was subjected to quality assessment using CheckM v1.0.5 (Parks et al., 2015) and Quast v4.5 (Gurevich et al., 2013). CheckM estimated the completeness and contiguity, while Quast was used to detect misassemblies, mismatches, and indels.

\section{Genome Analysis}

Genome assemblies were submitted to National Center for Biotechnology Information (NCBI) GenBank and annotated using the Prokaryotic Genome Annotation Pipeline (PGAP v.4.1) from NCBI (Tatusova et al., 2016). The resistance profile of the assembled genome sequences was obtained from Resfinder 4.1 available from CGE server (https://cge.cbs.dtu.dk/services/ ResFinder/). Similarly, the presence of plasmids in the genomes was identified and characterized using PlasmidFinder (v.1.3) available at CGE server (https://cge.cbs.dtu.dk/services/ PlasmidFinder). Furthermore, MLST and virulence locus (yersiniabactin, aerobactin, and other siderophore production systems) were identified using Kleborate (v.2.0.0) (https://github. com/katholt/Kleborate) (Lam et al., 2021). The presence of virulence factors was confirmed using virulence database at Pasteur Institute for Kp (https://bigsdb.pasteur.fr/cgi-bin/bigsdb/ bigsdb.pl?db=pubmlst_klebsiella_seqdef\&page=sequenceQuery). The final assembled circular chromosome and plasmid were visualized using CGview server v.1.0 (Grant and Stothard, 2008).

\section{Screening of Mutations in $r m p A$ and $r m p A 2$}

The sequences of $r m p A$ and $r m p A 2$ obtained from whole genome were compared with references AUB50662.1 and AAR07704.1, respectively, to determine the mutations. The sequences were aligned using Clustal Omega (https://www.ebi.ac.uk/Tools/msa/ clustalo/); and mismatches in nucleotides were identified. The allele numbers of $r m p A$ and $r m p A 2$ were assigned using the database at BIGSdb [Bacterial Isolate Genome Sequence Database] (https://bigsdb.pasteur.fr/cgi-bin/bigsdb/bigsdb.pl? $\mathrm{db}=$ pubmlst_klebsiella_seqdef\&page=sequenceQuery).

\section{In-Silico Methods Modeling of the Proteins}

The genomic study was subsequently correlated with structural profiles of RmpA, RmpA2, IutA, and IucA to obtain an insight into their structural stabilities. However, no crystal structures of RmpA, RmpA2, and IutA were available at present as observed from NCBI-BLASTp search in Protein Data Bank. Hence, intensive computational modeling and validations were employed to predict the structure of the proteins. The sequences of RmpA, RmpA2, IucA, and IutA as well as the mutant varieties of RmpA2 were 
obtained from our WGS data. In the absence of suitable templates, the entire structures of RmpA, RmpA2, and IutA were modeled using an extended dual-step method. In the first step, the $3 \mathrm{D}$ structure prediction (with available sequences) based on homology and threading method was primarily performed by using I-TASSER (https://zhanglab.ccmb.med.umich.edu/ITASSER/) and RaptorX (http://raptorx.uchicago.edu/) servers using their default parameters. From the server results, the topranked models were chosen based on C-scores, B-factors, and low root-mean-square deviation (RMSD) values. C-score ( -5 to 2 ) predicts the global structure accuracy when original structures are unavailable. The modeled proteins that possessed residue-level normalized B-factor trajectories around 0.00 for all structural patterns (helices, strands, and coils) were chosen (Yang and Zhang, 2015; Wang et al., 2016). In the second step of modeling, the server-based models were used as templates to model the final structures of RmpA, RmpA2, and IutA proteins using standalone python-based software MODELLER 10.0. The structures built with MODELLER satisfy spatial restraints expressed as probability density functions and merged with an optimized combination of conjugate gradients and molecular dynamics. This model-building procedure is identical to NMR spectroscopy-based structure elucidation (Webb and Sali, 2017). This extended method of computational modeling comprising homology, threading, and dynamics complied with vital structural parameters and hence minimized local structural errors to improve the quality of predicted model during the unavailability of structural templates (Basu et al., 2021).

Finally, for each of the steepest descent and conjugate gradient parameters, 2,000 steps were performed in Swiss PDB viewer (SPDBV) using GROMOS96 force field in vacuo to optimize the modeled structures (Kaplan and Littlejohn, 2001). The structure of IucA was retrieved from Protein Data Bank (ID: 5JM8), and missing residues were added using SPDBV. The sequence of IucA was further aligned with the IucA sequence obtained from WGS data and hence validated. The structures and graphs generated were retrieved from respective sites. The protein structures were visualized in UCSF-CHIMERA (Pettersen et al., 2004).

\section{Protein-Structure Validations}

The modeled RmpA, RmpA2, and IutA proteins were refined through the GalaxyRefine server (http://galaxy.seoklab.org/ index.html) to assess and improve the clash scores, poor rotamers, percentage Ramachandran outliers, and percentage bad side-chain rotamers (Heo et al., 2013). The final models were validated using HARMONY (for RmpA and RmpA2) (http://caps.ncbs.res.in/harmony/) and ProSA-web (for all three modeled proteins) (https://prosa.services.came.sbg.ac.at/ prosa.php) servers. HARMONY measures the substitution scores on an individual residue level to assess errors in the protein's 3D conformation based on folding patterns of previously characterized structures. The substitution graph from HARMONY provides the smoothened scores between query sequences in comparison with the reverse sequences of the respective proteins. The reverse sequence and its scores are used as a control to identify local errors in the proposed protein model. The query sequence should have a higher substitution score than the reverse sequence to avoid possible local errors (Pugalenthi et al., 2006). ProSA-web highlighted protein structure errors based on energy plots and Z-score derived from conformational variations concerning experimentally derived structural patterns (Wiederstein and Sippl, 2007).

\section{Protein Backbone Stability Analysis}

The backbone stability of the proteins was performed using server DynaMine (http://dynamine.ibsquare.be/). DynaMine predicts backbone flexibility at the residue level in the form of backbone $\mathrm{N}-\mathrm{H} \mathrm{S}^{2}$-order parameter values, which were directly determined from experimental NMR chemical shifts. This $S^{2}$ values portray the restrictions atomic bond movements with respect to the molecular reference frame. A value of 1 means stable conformation (high rigidity), while a value of 0 means fully random bond vector movement (highly dynamic). Furthermore, values $>0.8$ are referred to as considerably rigid, between 0.6 and 0.7 may depict functionally contextual, and $<0.6$ denotes highly flexible backbone. DynaMine uses a simple linear regression method to accurately distinguish between folded domains and disordered regions of different magnitudes (Cilia et al., 2014). The average $S^{2}$ scores of the whole proteins and their respective functional domains were determined. The functional domains of the proteins were validated through INTERPRO and Pfam servers.

\section{Residue-Level Propensity Analysis Through Coarse Dynamics}

CABSflex server (http://biocomp.chem.uw.edu.pl/CABSflex2/) was employed for coarse dynamics study to depict the residue-level fluctuations when compared with the most favorable conformation of the protein. The stabilities were analyzed from the root-meansquare fluctuation (RMSF) values generated based on default restraint parameters. The restraints provided maximum and minimum ranges to pair atoms and contain them within defined spaces with their dynamic orientations. The deviations beyond assigned ranges were designated as unstable. The default settings and restraints were optimized to merge coarse dynamics simulations and consensus protein fluctuations in aqueous environment derived by all-atom molecular dynamics simulation (10-ns timescale with suitable force fields). The default parameters were selected with gap $=3$ (minimum distance between previous and next amino acid in the chain to be restrained); minimum and maximum conformational distances were 3.8 and $8.0 \AA$, respectively (Jamroz et al., 2013).

\section{RESULTS}

\section{Phenotypic Characterization of Klebsiella pneumoniae and Clinical Details of the Patients}

All the $27 \mathrm{MDR} \mathrm{Kp}$ included in the study were resistant to ceftazidime, cefepime, meropenem, imipenem, amikacin, ciprofloxacin, and minocycline as determined by disc diffusion. Since the isolates were resistant to meropenem and imipenem, 
they are referred to as carbapenem-resistant (CR-hvKp). Eleven isolates (41\%) retained intermediate susceptibility to colistin with MIC of $\leq 2 \mu \mathrm{g} / \mathrm{ml}$. Twenty $\mathrm{Kp}$ isolates in the study were string test negative, lacking hypermucoviscosity, due to frameshift mutations in $r m p A$ and $r m p A 2$ as mentioned in Table 1.

Among the study population, 16 patients developed hospitalacquired CR-hvKp infection, while three acquired communityassociated infection. The nature of infection in the other eight patients could not be established from electronic medical records. A combination of colistin and tigecycline was most frequently used to treat these CR-hvKp infections ( $\mathrm{n}=11,44 \%)$. The other antimicrobials that were used in combination therapy include meropenem with colistin/tigecycline and metronidazole. Twelve patients with CR-hvKp had a fatal outcome, and the mean duration of stay in the hospital was 91 days among this cohort. Besides, half of the study population were neutropenic and immunosuppressed.

\section{Genomic Characterization of Carbapenem Resistant Hypervirulent Klebsiella pneumoniae}

The genomic characteristics of the CR-hvKp are detailed in Table 1. The virulence plasmid present in the isolates predominantly carried IncHI1B (pNDM-MAR) backbone. As shown in Table 1, most of the CR-hvKp belonged to international high-risk clones such as ST11, ST15, and ST43, which carry ARGs on multiple plasmids.

Interestingly, there was a single CR-hvKp belonging to ST23 that carried both $r m p A$ and $r m p A 2$ on the virulence plasmid. In addition, it carried the carbapenemase-encoding gene, $b l a_{\mathrm{OXA}}$ 232, on ColKP3 plasmid. When compared with other study isolates, notably, this isolate carried additional virulence factors such as colibactin, salmochelin, and those for allantoin metabolism ( $h y i, g l x K, g l x R, y b b W, y b b Y, y l b E$, and $y l b F)$. The other isolates are briefly described below and carry diverse $\mathrm{K}$ and $\mathrm{O}$ antigens depending on the clonal group they belong to.

\section{CG11}

Eight isolates belonged to CG11, of which seven belonged to ST11, while a single isolate was of ST3791, a single-locus variant of ST11. All the isolates of CG11 belonged to capsule type, K24, with $\mathrm{O} 2 \mathrm{v} 1 \mathrm{O}$ antigen. These isolates coded for yersiniabactin (ybt16) mobilized by ICEKp12. The isolates lacked $r m p A$ but carried $r m p A 2$. Isolates of CG11 carried $b l a_{\mathrm{NDM}}$ and $b l a_{\mathrm{OXA}-232}$ alone or in combination. Seven isolates in this group were resistant to colistin and carried two amino acid substitutions in pmrB (T246A and R256G) as shown in Table 1. The other mutations conferring colistin resistance include frameshift in phoQ in the isolate belonging to ST3791.

\section{CG43}

Five isolates belonging to ST43 and one of ST3790, a single-locus variant of ST43, were identified. All the six isolates carried a frameshifted rmpA2 in the virulence plasmid. ST43 isolates carried $y b t-9$ on ICEKp3, while the isolate belonging to ST3790 carried $y b t$ of unknown type. The isolates belonged to K30 with
O1v1 antigen. Isolates of CG43 lacked $b l a_{\mathrm{NDM}}$ and predominantly carried $b l a_{\mathrm{OXA}-181}$, except one isolate that


resistant to colistin due to single amino acid substitutions in pmrA (A217V) and pmrB (T246A) as mentioned in Table 1. K. pneumoniae ST3790, in addition to the mutations in $p m r A$ and $p m r B$, showed deletion of $m g r B$, which confers resistance to colistin.

\section{ST231}

Six isolates belonging to ST231, although lacked the rmpA2, carried aerobactin, one of the key virulence factors. In two isolates, aerobactin was identified on a plasmid with IncFIAIncFII replicons (data not shown). However, for the other isolates since complete genomes were not available, it was impossible to determine if aerobactin was inserted into chromosome or another plasmid excluding the virulence plasmid. These isolates carried bla $a_{\mathrm{OXA}-232}$ mobilized by ColKP3 plasmid. Figure 1 shows the virulence plasmid (carrying aerobactin) in isolate B6753 (CP067046) compared with the reference virulence plasmid SGH10 (CP025080) and the closest-matching plasmids CP045675 and CP052259. The virulence plasmid of B6753 carries integron class 1 , which is absent in the other three plasmids. Heavy metal resistance genes such as those coding for copper ( $p c o)$, silver ( $i l$ ), tellurium (ter), and mercury (mer) are also shown in Figure 1. A single isolate in this group was non-susceptible to colistin due to truncated $m g r B$ of eight amino acids (Table 1).

\section{Other Clones}

Three CR-hvKp belonged to ST15, while a single isolate each belonged to ST86 and ST4847 (TLV of ST29). Two isolates of ST15 were non-susceptible to colistin but showed different chromosomal mutations (Table 1). Interestingly, an isolate belonging to ST15 carried the K2 capsule type, which is in contrast to the present knowledge of $\mathrm{K} 2$ antigen being confined to hvKp clones such as ST65 and ST86. This isolate also showed the presence of frameshift mutation in rmpA2. The isolate belonging to ST86 was associated with K2 antigen, a characteristic feature of hvKp, which is also reported by other studies. ST86 K. pneumoniae in this study also carried nonfunctional $m g r B$ due to premature stop codon in the third amino acid.

Among the other factors contributing to virulence, the siderophore salmochelin was present in a single isolate each of ST15, ST86, ST231, and ST4847. Interestingly, the CRKp in the present study have acquired the virulence plasmid with $r m p A 2$, while $r m p A$ was present in three isolates only. In addition, aerobactin, present in all the isolates, was the other promising molecular marker among these isolates.

\section{RmpA2 Variants}

Among the study isolates, six $r m p A 2$ variants were identified as shown in Figure 2. Variants 2-6 were truncated at various positions, while rmpA2_9 had a single amino acid substitution at position 101 wherein glutamine is replaced by lysine. The wild-type $\operatorname{rmp} A 2$, encoded by 212 amino acids, has the signal transduction response regulator region from 131 to 196 amino 
TABLE 1 | Results of the whole-genome analysis showing characteristics of carbapenem resistant hypervirulent Kp.

\begin{tabular}{|c|c|c|c|c|c|c|c|c|c|c|}
\hline Accession number & $\begin{array}{l}r m p A \\
\text { and/or } \\
r m p A 2\end{array}$ & $\begin{array}{l}\text { String } \\
\text { test }\end{array}$ & $\begin{array}{l}\text { Capsule } \\
\text { type }\end{array}$ & $\begin{array}{c}\text { O } \\
\text { antigen }\end{array}$ & ST & $\begin{array}{c}y b t, \\
\text { ICEKp }\end{array}$ & Resistance genes & $\begin{array}{l}\text { Colistin } \\
\text { MIC and } \\
\text { resistance } \\
\text { mechanism }\end{array}$ & Plasmids & Virulence genes \\
\hline JACWMO000000000 & $\begin{array}{l}\text { Both } \\
(r m p A 2-5)\end{array}$ & pos & $\mathrm{K} 1$ & O1v2 & 23 & $\begin{array}{l}\text { ybt8; } \\
\text { ICEKp3 }\end{array}$ & bla ${ }_{\mathrm{OXA}-232}$, bla $\mathrm{SHV}-11_{1}$, fOSA & $\mathrm{MIC} 1 \mu \mathrm{g} / \mathrm{ml}$ & $\begin{array}{l}\text { IncHI1B } \\
\text { (pNDM-MAR) } \\
\text { \#, ColKP3 }\end{array}$ & $\begin{array}{l}\text { allABDRS, fyuA, hyi, } \\
\text { irp1, irp2, colibactin, } \\
\text { fdrA, gcl, glxK, glxR, } \\
\text { aerobactin, } \\
\text { salmochelin, ybbW, } \\
\text { ybbY, ylbE, ylbF, } \\
\text { mrkABCDFHIJ }\end{array}$ \\
\hline MNPB00000000 & $\begin{array}{l}r m p A 2 \\
(2)\end{array}$ & neg & K24 & O2v1 & 11 & $\begin{array}{l}\text { ybt16; } \\
\text { ICEKp12 }\end{array}$ & 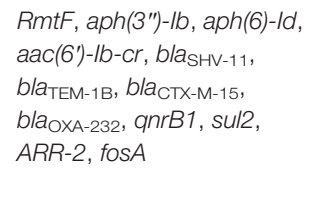 & $\begin{array}{l}\mathrm{D} 150 \mathrm{H}, \\
\mathrm{T} 246 \mathrm{~A}, \\
\mathrm{R} 256 \mathrm{G} \text { in } \\
\text { pmrB }(8 \mu \mathrm{g} / \\
\mathrm{ml})\end{array}$ & $\begin{array}{l}\text { IncFIIK, } \\
\text { IncFIB(pQil), } \\
\text { IncHI1B } \\
\text { (pNDM-MAR) } \\
\text { \#, ColKP3, } \\
\text { IncR }\end{array}$ & $\begin{array}{l}\text { fyuA, irp1, irp2, } \\
\text { mrkABCDFHIJ, } \\
\text { aerobactin }\end{array}$ \\
\hline MNPC00000000 & $\begin{array}{l}r m p A 2 \\
(4)\end{array}$ & neg & K24 & O2v1 & 11 & $\begin{array}{l}\text { ybt16; } \\
\text { ICEKp12 }\end{array}$ & $\begin{array}{l}\text { RmtF, aph(3")-lb, aph(6)-ld, } \\
\text { aac(6')-lb-cr, bla } \\
\text { bla }_{\mathrm{TEM}-1 \mathrm{~B}-11}, \\
\text { bla }_{\mathrm{OXA}-232}, \text { anrB1, sul2, } \\
\text { ARR-2, fosA }\end{array}$ & $\begin{array}{l}\mathrm{D} 150 \mathrm{H}, \\
\mathrm{T} 246 \mathrm{~A}, \\
\mathrm{R} 256 \mathrm{G} \text { in } \\
\text { pmrB }(8 \mu \mathrm{g} / \\
\mathrm{ml})\end{array}$ & $\begin{array}{l}\text { IncFIIK, } \\
\text { IncFIB(pQil), } \\
\text { IncHI1B } \\
\text { (pNDM-MAR) } \\
\text { \#, ColKP3, } \\
\text { IncR }\end{array}$ & $\begin{array}{l}\text { fyuA, irp1, irp2, } \\
\text { mrkABCDFHIJ, } \\
\text { aerobactin }\end{array}$ \\
\hline MNPG00000000 & $\begin{array}{l}r m p A 2^{*} \\
(2)\end{array}$ & neg & K24 & O2v1 & 11 & $\begin{array}{l}\text { ybt16; } \\
\text { ICEKp12 }\end{array}$ & 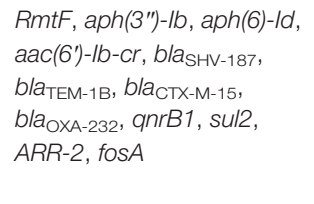 & $\begin{array}{l}\mathrm{D} 150 \mathrm{H}, \\
\mathrm{T} 246 \mathrm{~A}, \\
\mathrm{R} 256 \mathrm{G} \text { in } \\
\text { pmrB }(8 \mu \mathrm{g} / \\
\mathrm{ml})\end{array}$ & $\begin{array}{l}\text { IncFIIK, } \\
\text { IncFIB(pQil), } \\
\text { IncHI1B } \\
\text { (pNDM-MAR) } \\
\text { \#, ColKP3, } \\
\text { IncR }\end{array}$ & $\begin{array}{l}\text { fyuA, mrkABCDFHIJ, } \\
\text { aerobactin }\end{array}$ \\
\hline MCFQ00000000 & $\begin{array}{l}r m p A 2 \\
(2)\end{array}$ & neg & K24 & O2v1 & 11 & $\begin{array}{l}\text { ybt16; } \\
\text { ICEKp12 }\end{array}$ & 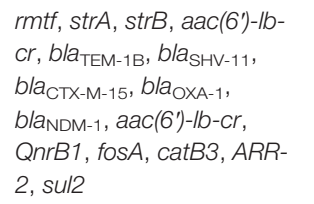 & $\begin{array}{l}\mathrm{MIC} \leq 0.12 \\
\mu \mathrm{g} / \mathrm{ml}\end{array}$ & $\begin{array}{l}\text { IncFIB(pQII), } \\
\text { IncHI1B } \\
\text { (pNDM-MAR) } \\
\#, \text { IncFIIK, } \\
\text { IncR }\end{array}$ & $\begin{array}{l}\text { fyuA, irp2, aerobactin, } \\
\text { mrkABCDFHIJ }\end{array}$ \\
\hline SRS3894081 & $\begin{array}{l}r m p A 2 \\
\text { (9) }\end{array}$ & pos & K24 & O2v1 & 11 & $\begin{array}{l}\text { ybt16; } \\
\text { ICEKp12 }\end{array}$ & 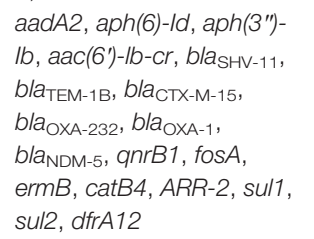 & $\begin{array}{l}\text { T246A, } \\
\text { R256G in } \\
\text { pmrB (128 } \\
\mu \mathrm{g} / \mathrm{ml})\end{array}$ & $\begin{array}{l}\text { IncFIB(pQil), } \\
\text { IncFIl, } \\
\text { IncFIIK, } \\
\text { IncHI1B } \\
\text { (pNDM-MAR) } \\
\text { \#, ColKP3, } \\
\text { IncR }\end{array}$ & $\begin{array}{l}\text { fyuA, irp2, } \\
\text { mrkABCDFHIJ, } \\
\text { aerobactin }\end{array}$ \\
\hline SRS3894067 & $\begin{array}{l}r m p A 2 \\
(2)\end{array}$ & neg & K24 & O2v1 & 11 & $\begin{array}{l}\text { ybt16; } \\
\text { ICEKp12 }\end{array}$ & 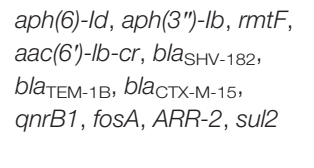 & $\begin{array}{l}\text { P95L, } \\
\text { T246A, } \\
\text { R256G in } \\
p m r B(64 \\
\mu \mathrm{g} / \mathrm{ml})\end{array}$ & $\begin{array}{l}\text { IncHI1B } \\
\text { (pNDM-MAR) } \\
\#, \text { IncFIB } \\
\text { (pQil), IncR, } \\
\text { IncFIIK }\end{array}$ & $\begin{array}{l}\text { fyuA, irp1, irp2, } \\
\text { mrkABCDFHIJ, } \\
\text { aerobactin }\end{array}$ \\
\hline SRS3894082 & $\begin{array}{l}r m p A 2 \\
\text { (9) }\end{array}$ & neg & K24 & $\mathrm{O} 2 \mathrm{v} 1$ & 11 & $\begin{array}{l}\text { ybt16; } \\
\text { ICEKp12 }\end{array}$ & 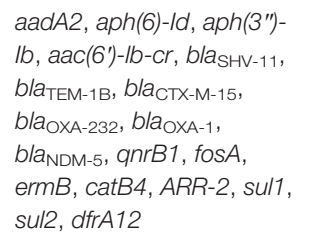 & $\begin{array}{l}\text { T246A, } \\
\text { R256G in } \\
\text { pmrB (128 } \\
\mu \mathrm{g} / \mathrm{ml})\end{array}$ & $\begin{array}{l}\text { IncFIB(pQil), } \\
\text { IncFIl, } \\
\text { IncFIIK, } \\
\text { IncHI1B } \\
\text { (pNDM-MAR) } \\
\text { \#, ColKP3, } \\
\text { IncR }\end{array}$ & $\begin{array}{l}\text { fyuA, irp1, } \\
\text { mrkABCDFHIJ, } \\
\text { aerobactin }\end{array}$ \\
\hline MNPD00000000 & $\begin{array}{l}r m p A 2^{*} \\
\text { (2) }\end{array}$ & neg & K24 & $\mathrm{O} 2 \mathrm{v} 1$ & $\begin{array}{l}3791 \\
\text { (SLV } \\
\text { of } \\
\text { ST11) }\end{array}$ & $\begin{array}{l}\text { ybt16; } \\
\text { ICEKp12 }\end{array}$ & $\begin{array}{l}R m t F, \text { aph(3")-lb, aph(6)-ld, } \\
\text { aac(6')-lb-cr, bla TEM-1B, } \\
\text { bla } \mathrm{CTX-M-15,} \mathrm{qnrB1,} \mathrm{sul2,} \\
\text { ARR-2, fosA }\end{array}$ & $\begin{array}{l}\text { frameshifted } \\
\text { phoQ; } \\
\text { D150H, } \\
\text { T246A, } \\
\text { R256G in } \\
\text { pmrB (8 } \mu \mathrm{g} / \\
\mathrm{ml})\end{array}$ & $\begin{array}{l}\text { IncFIIK, } \\
\text { IncFIB(pQil), } \\
\text { IncHI1B } \\
\text { (pNDM-MAR) } \\
\text { \#, IncR }\end{array}$ & $\begin{array}{l}\text { fyuA, irp1, irp2, } \\
\text { mrkABCDFHIJ, } \\
\text { aerobactin }\end{array}$ \\
\hline MCFP00000000 & $\begin{array}{l}r m p A 2 \\
(9)\end{array}$ & pos & K30 & $\begin{array}{l}\mathrm{O} 1 / \\
\mathrm{O} 2 \mathrm{v} 1\end{array}$ & 43 & $\begin{array}{l}\text { ybt9, } \\
\text { ICEKp3 }\end{array}$ &  & $\begin{array}{l}\mathrm{MIC} 0.25 \\
\mu \mathrm{g} / \mathrm{ml}\end{array}$ & $\begin{array}{l}\text { IncFIB(pQII), } \\
\text { IncHI1B } \\
\text { (pNDM-MAR) }\end{array}$ & $\begin{array}{l}\text { fyuA, irp1, irp2, kfuA, } \\
m r k H, m r k l \\
\text { aerobactin }\end{array}$ \\
\hline
\end{tabular}


TABLE 1 | Continued

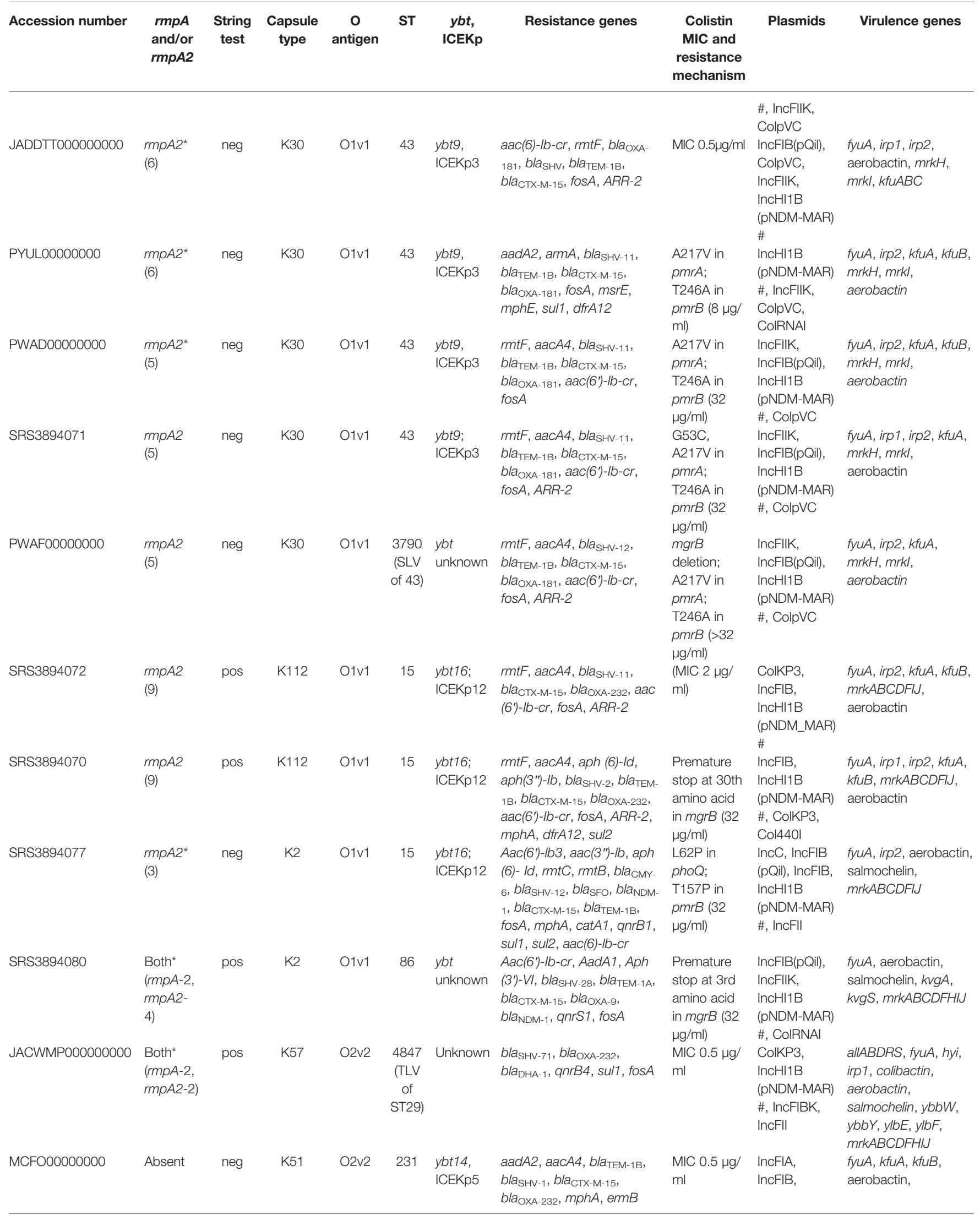


TABLE 1 | Continued

\begin{tabular}{|c|c|c|c|c|c|c|c|c|c|c|}
\hline Accession number & $\begin{array}{l}r m p A \\
\text { and/or } \\
r m p A 2\end{array}$ & $\begin{array}{c}\text { String } \\
\text { test }\end{array}$ & $\begin{array}{c}\text { Capsule } \\
\text { type }\end{array}$ & $\begin{array}{c}\text { O } \\
\text { antigen }\end{array}$ & ST & $\begin{array}{c}y b t, \\
\text { ICEKp }\end{array}$ & Resistance genes & $\begin{array}{l}\text { Colistin } \\
\text { MIC and } \\
\text { resistance } \\
\text { mechanism }\end{array}$ & Plasmids & Virulence genes \\
\hline SGIY00000000 & Absent & neg & K51 & O1v2 & 231 & $\begin{array}{l}\text { ybt14; } \\
\text { ICEKp5 }\end{array}$ &  & $\begin{array}{l}\text { Premature } \\
\text { stop codon } \\
\text { at the 9th } \\
\text { amino acid } \\
\text { in } m g r B ~(>4 \\
\mu \mathrm{g} / \mathrm{ml})\end{array}$ & $\begin{array}{l}\text { ColKp3, } \\
\text { IncFIIK, IncFII } \\
\text { ColKP3, } \\
\text { IncFIA, } \\
\text { IncFIB, } \\
\text { IncFIB(pQil), } \\
\text { IncFII, IncFIIK }\end{array}$ & $\begin{array}{l}\text { salmochelin, } \\
\text { mrkABCDFHIJ } \\
\text { fyuA, irp1, irp2, kfuA, } \\
\text { kfuB, mrkBCDFHIJ, } \\
\text { aerobactin }\end{array}$ \\
\hline JAAEYE000000000 & Absent & neg & K51 & O1v2 & 231 & $\begin{array}{l}\text { ybt14; } \\
\text { ICEKp5 }\end{array}$ & 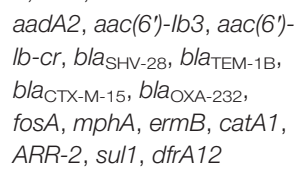 & $\begin{array}{l}\mathrm{MIC} \leq 1 \mu \mathrm{g} / \\
\mathrm{ml}\end{array}$ & $\begin{array}{l}\text { ColKP3, } \\
\text { IncFIA, } \\
\text { IncFIB(pQil), } \\
\text { IncFII, IncFIIK }\end{array}$ & $\begin{array}{l}\text { fyuA, irp1, irp2, kfuA, } \\
\text { kfuB, mrkBCDFHIJ, } \\
\text { aerobactin }\end{array}$ \\
\hline JAHPLH000000000 & Absent & neg & K51 & O1v2 & 231 & $\begin{array}{l}\text { ybt14; } \\
\text { ICEKp5 }\end{array}$ & $\begin{array}{l}\text { aadA2, rmtF, bla }{ }_{\mathrm{SHV}-1}, \\
\text { bla } \\
\text { mphA-1D, bla } \mathrm{OXA-232}, \\
\text { dfrA12 }\end{array}$ & $\begin{array}{l}\mathrm{MIC} \leq 0.25 \\
\mu \mathrm{g} / \mathrm{ml}\end{array}$ & $\begin{array}{l}\text { ColKP3, } \\
\text { IncFIA, } \\
\text { IncFIB(pQil), } \\
\text { IncFIIK, IncFII }\end{array}$ & $\begin{array}{l}\text { fyuA, irp1, irp2, } \\
\text { aerobactin, } \\
\text { salmochelin, } \\
\text { mrkABCDFHIJ }\end{array}$ \\
\hline JAHPLI000000000 & Absent & neg & K51 & O1v2 & 231 & $\begin{array}{l}\text { ybt14; } \\
\text { ICEKp5 }\end{array}$ & 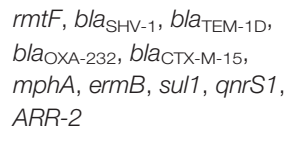 & $\begin{array}{l}\mathrm{MIC} \leq 0.5 \\
\mu \mathrm{g} / \mathrm{ml}\end{array}$ & $\begin{array}{l}\text { ColKP3, } \\
\text { IncFIA, } \\
\text { IncFIB(pQil), } \\
\text { IncFIIK, } \\
\text { CoIRNAI }\end{array}$ & $\begin{array}{l}\text { fyuA, irp1, irp2, } \\
\text { aerobactin, kfuABC, } \\
\text { mrkBCDFHIJ }\end{array}$ \\
\hline JAARNY000000000 & Absent & neg & K51 & O1v2 & 231 & $\begin{array}{l}\text { ybt14; } \\
\text { ICEKp5 }\end{array}$ & 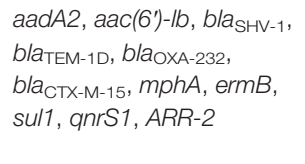 & $\begin{array}{l}\text { MIC } 0.5 \mu \mathrm{g} / \\
\mathrm{ml}\end{array}$ & $\begin{array}{l}\text { ColKP3, } \\
\text { IncFIA, } \\
\text { IncFIB(pQil), } \\
\text { IncFIIK, } \\
\text { Col440| }\end{array}$ & $\begin{array}{l}\text { fyuA, iroB, irp1, irp2, } \\
\text { kfuABC, aerobactin, } \\
\text { clbA, mrkABCDFHIJ }\end{array}$ \\
\hline
\end{tabular}

Colistin resistance amino acid substitutions: $P$, proline; L, leucine; $T$, threonine; $A$, alanine; $R$, arginine; $G$, glycine; $D$, aspartic acid; $V$, valine; ybt, Yersiniabactin.

ST, sequence type; SLV, single-locus variant; TLV, triple-locus variant; MIC, minimum inhibitory concentration; Kp, Klebsiella pneumoniae.

${ }^{*}$ Frameshift mutation was observed in rmpA2; variants of rmpA and rmpA2 are mentioned in brackets.

Virulence plasmid.

$\wedge$ Mutation in rmpA.

acids. This region is disrupted in the variants leading to a functional loss, which reflects as a lack of hypermucoviscous phenotype. Hence, the CR-hvKp cannot be screened using a positive string test.

\section{Protein Modeling and Structural Validations}

Based on Z-scores from ProSA-web signifying the overall quality of the modeled RmpA, RmpA2, and IutA proteins, it was observed that all three are well-poised among experimentally determined (NMR and X-ray diffraction) protein structures (Figure 3A). The residue-level substitution scores of the validated structures of RmpA, RmpA2, and IutA proteins as per the HARMONY calibration plot are considerably higher than the respective reverse sequences (control) designating minimum errors in terms of misfolded conformations (Figures 3B-D). After final refinement, the modeled structures possessed $>95 \%$ residues in Ramachandran favored region with low values corresponding to poor rotamers $(<1.5)$, which signify the conformational integrity of the protein structures.

The final predicted structures of RmpA, RmpA2, and IutA were submitted to Protein Model Database (PMDB) (http:// srv00.recas.ba.infn.it/PMDB/) having PMDB-IDs PM0083529,
PM0084102, and PM0084103. The final structures of all the classical proteins are shown in Figures 4A-D.

\section{Backbone Stability of the Proteins}

Figures 5A, B show that all the proteins have a stable backbone conformation [high average rigidity of backbone $\rightarrow S^{2}>0.8$ ], conferring structural integrity to the proteins.

The functionally significant domains of IucA are aerobactinsiderophore biosynthesis domain (between residues 138 and 361, average $S^{2}$ score $=0.80$ ) and ferric-ion reductase domain (between residues 400 and 556; average $S^{2}$ score $=0.88$ ). The characteristic membrane TonB-dependent receptor domain of IutA is located between residues 238 and 730 (average $S^{2}$ score $=0.82$ ), and a smaller plug domain is located between residues 45 and 146 (average $S^{2}$ score $=0.79$ ). The signal transduction regulator domain (STRD) of RmpA2 is located between amino acid residues 131 and 196 (average $S^{2}$ score $=0.87$ ). The average $S^{2}$ scores indicate that both IutA and IucA have high backbone rigidity in their functional domains as well as the entire structure [overall $0.80 \pm 0.02 \AA]$. Hence, they have stable structure-function profiles. The overall backbone rigidity of $\operatorname{RmpA} 2(0.88 \pm 0.006 \AA)$ is marginally higher than that of IutA and IucA. Hence, classical RmpA2 has better backbone stability, which supported its previous consideration as a molecular marker for hvKp (Russo et al., 2018). 


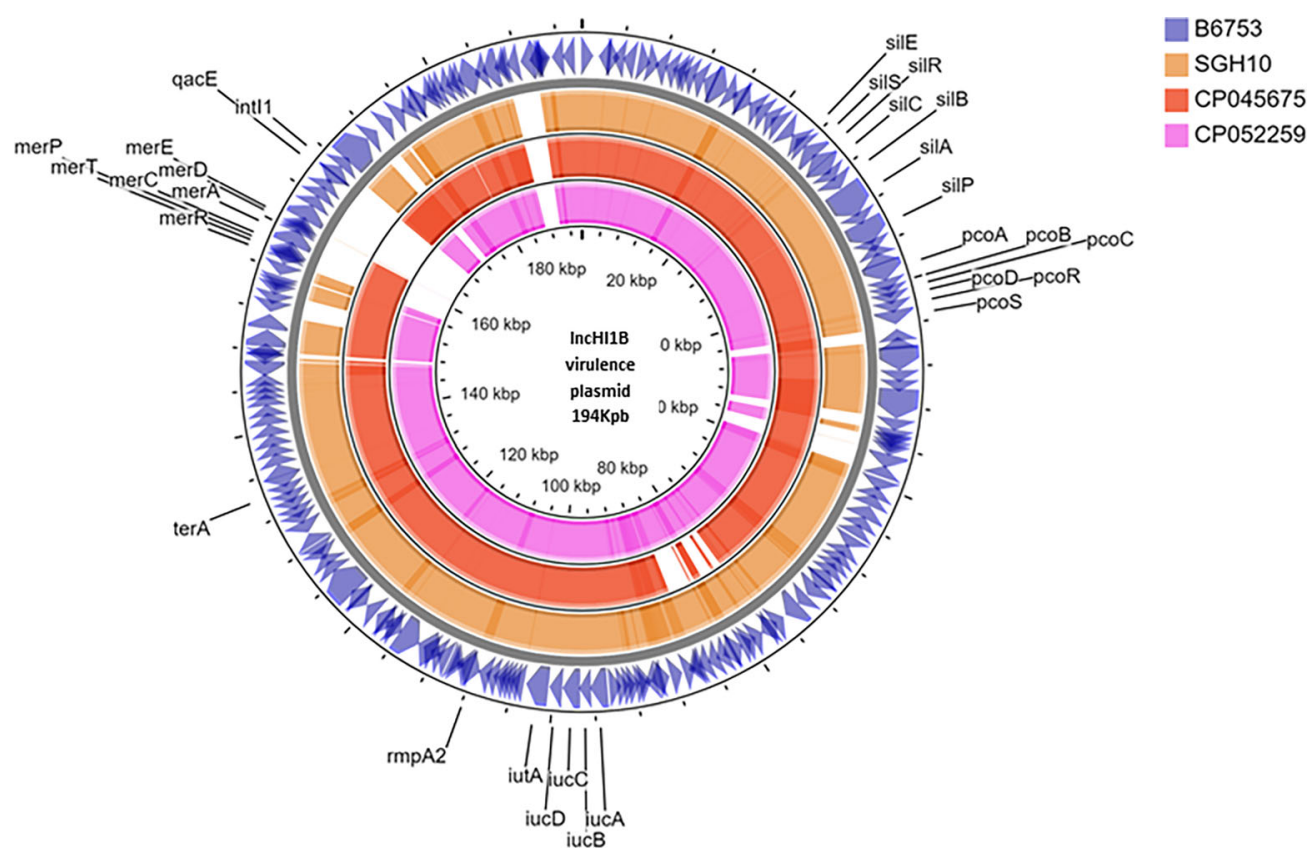

FIGURE 1 | Circular representation of virulence plasmid assembled from Kp B6753 displayed using CG view server. Comparison of virulence plasmid carrying rmpA2 among (1) Kp strain B6753 (CP067046) from ST43 (2) Kp strain SGH10 (CP025080) from ST23 (3) Kp strain WSD411 (CP045675) from ST15 (4) Kp strain E16KP0290 (CP052259) from ST65.

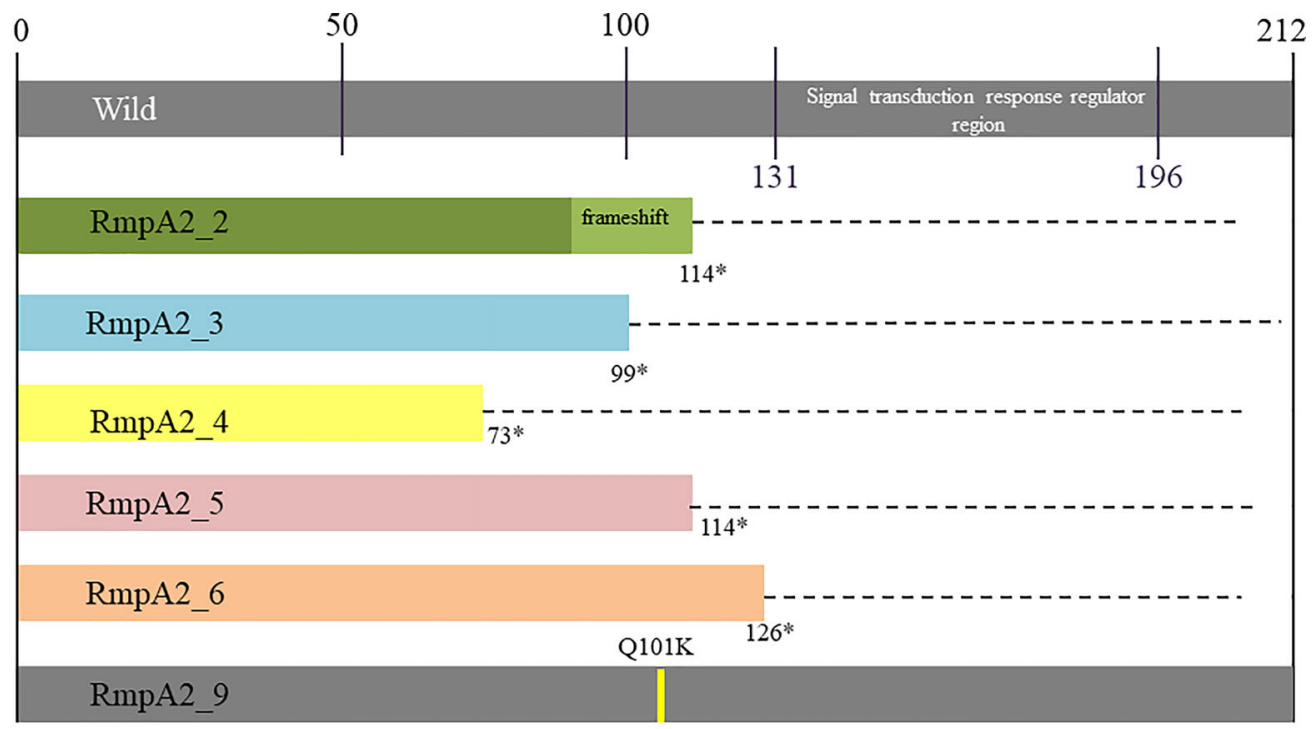

*truncation at various positions

FIGURE 2 | Variants of RmpA2 observed among carbapenem resistant hypervirulent Kp from India..

However, owing to indel mutations in our isolates between nucleotides 285 and 290, major frameshifts were observed in the backbone trajectory of RmpA2 variants (Figure 5C) when compared with the classical RmpA2. The mutant RmpA2_3 showed identical trajectory when compared with the classical counterpart, although there was an upstream shift of the STRD. The mutant RmpA2_4 portrayed a slightly different frameshift than the rest owing to an insertion mutation at nucleotide position 197. 




FIGURE 3 | Global model qualities of RmpA, RmpA2 and lutA with respect to (A) experimentally derived structures and (B-D) residue-level substitution scores signifying local folding conformations.

All of the mutants of RmpA2 were observed to have major reductions $S^{2}$ scores, especially in the SRTD domain (by $\sim 40 \%$ ) converting the structural profile of this region from a stably rigid domain to a considerably flexible one owing to upstream truncations. These changes can potentially contribute to the under-functioning of RmpA2 in the variants, thereby failing to express hypermucoviscous phenotype. However, no such truncations/indel mutations were observed in IutA or IucA, and they expressed a uniformly stable profile in all the isolates.

\section{Coarse Dynamics Analysis to Assess the Structural Stability Profile}

The relative stability of RmpA, RmpA2, IutA, and IucA analyzed using CABSflex server showed that that the relative fluctuations of RmpA are higher than those of RmpA2 (Figure 6A), although IutA and IucA possessed similar dynamicity (Figure 6B). From the conformational perspective, it can be inferred that classical RmpA2 possesses a better stability profile than RmpA as well as IucA and IutA, which supports the backbone dynamicity of the 
A
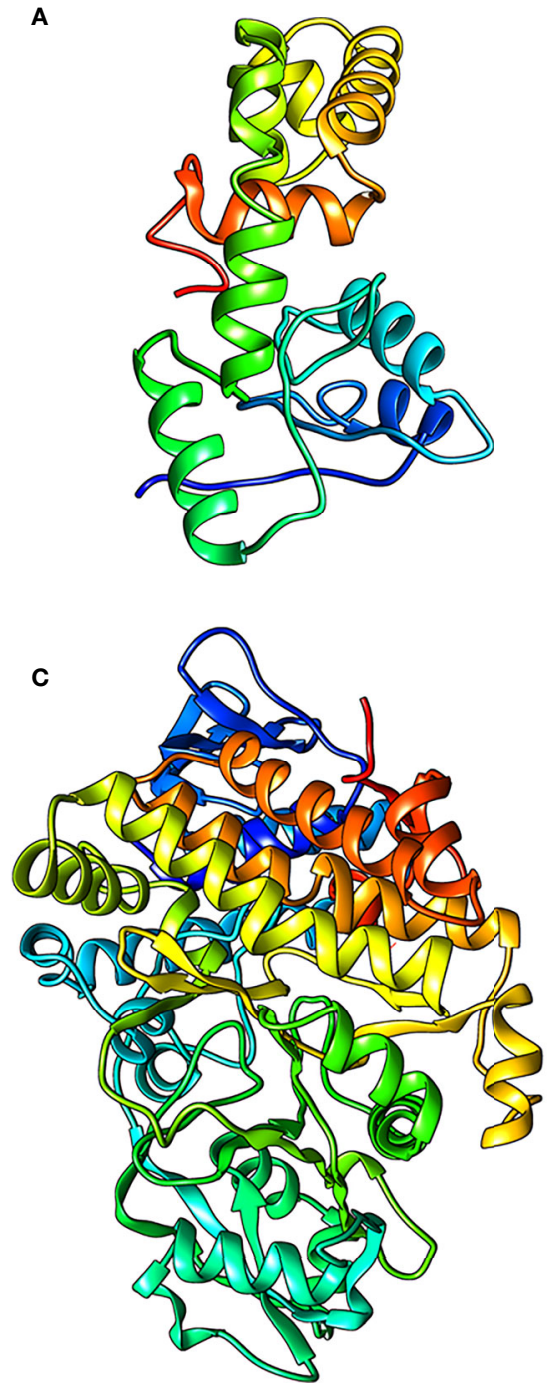

B

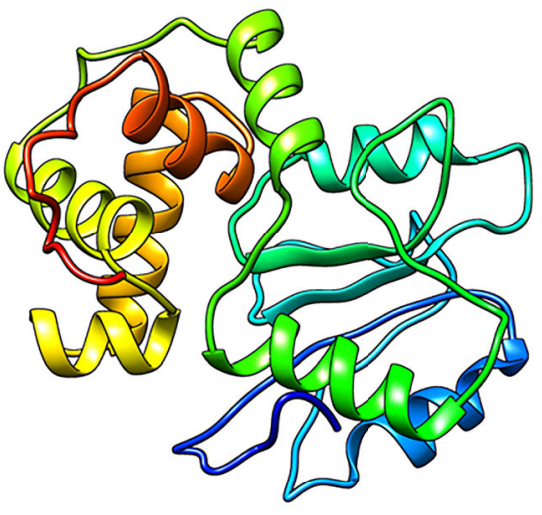

D

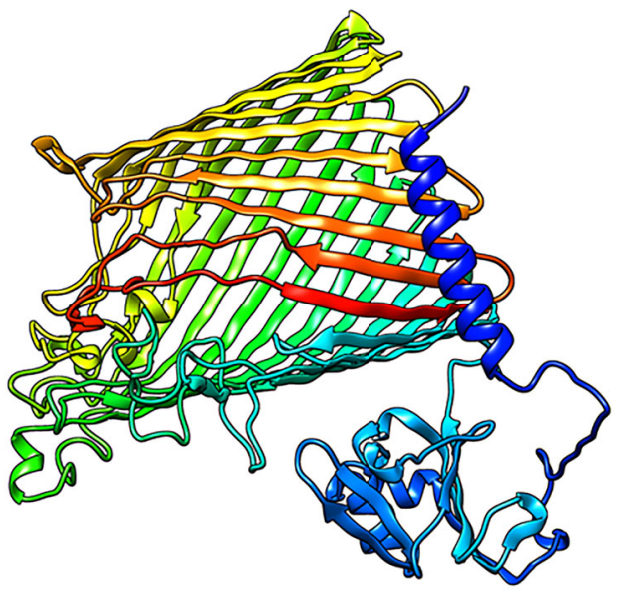

FIGURE 4 | The refined structures of the proteins (A) RmpA (B) RmpA2 (C) lucA and (D) lutA.

proteins. Furthermore, the average RMSF of the residues in signal regulatory region of RmpA2 (residues between 131 and $196)$ is lower $(0.59 \AA)$ than the average RMSF of the transcription regulatory residues (residues between 109 and 156) in RmpA $(0.91 \AA)$. This portrays a better structure-function stability profile of RmpA2 over RmpA and hence is predominantly present among CR-hvKp. The average RMSF of the functional domain residues in aerobactin such as the aerobactin-synthetase domain (IucA) and ferric-ion reductase (IutA) domain is 0.79 and $0.77 \AA$, respectively. The receptor domain (residues 338730 ) of IutA has fairly lower fluctuations (average RMSF $=0.77$ $\AA$ ), similar to IucA. This portrays a better structure-function stability profile of aerobactin proteins over RmpA, but not RmpA2. However, in truncated RmpA2 proteins, the resultant RMSF values are very high (and irregular), which do not follow a pattern as compared with the parent RmpA2 protein (not shown).

\section{In-Vitro Fitness of Hypervirulent Klebsiella pneumoniae}

Growth curve was obtained for eight hypervirulent $K$. pneumoniae, which is shown in Figure 7. An isolate belonging to ST2096, carrying truncated rmpA2 (JAAQSY000000000), and an isolate with wild-type $r m p A 2$ belonging to ST23 (MBFY00000000) isolated at the study center were used to compare the growth rate of other CR-hvKp in the present study. Three isolates carrying aerobactin without $r m p A / r m p A 2$ belonging to ST231 showed a similar growth rate as compared with a pan-susceptible ST23 isolate that carried wild-type $r m p A$ and $r m p A 2$. The isolates belonging to high-risk clones ST11 and ST15 with truncated rmpA2 grew slower than ST23 and other isolates carrying aerobactin alone (ST231) as shown in Figure 7. ST11 MDR-hvKp carried rmpA2_2 truncated to 114 amino acids, while ST15 MDR-hvKp carried rmpA2_9 with a single amino acid substitution. However, these findings need to be 

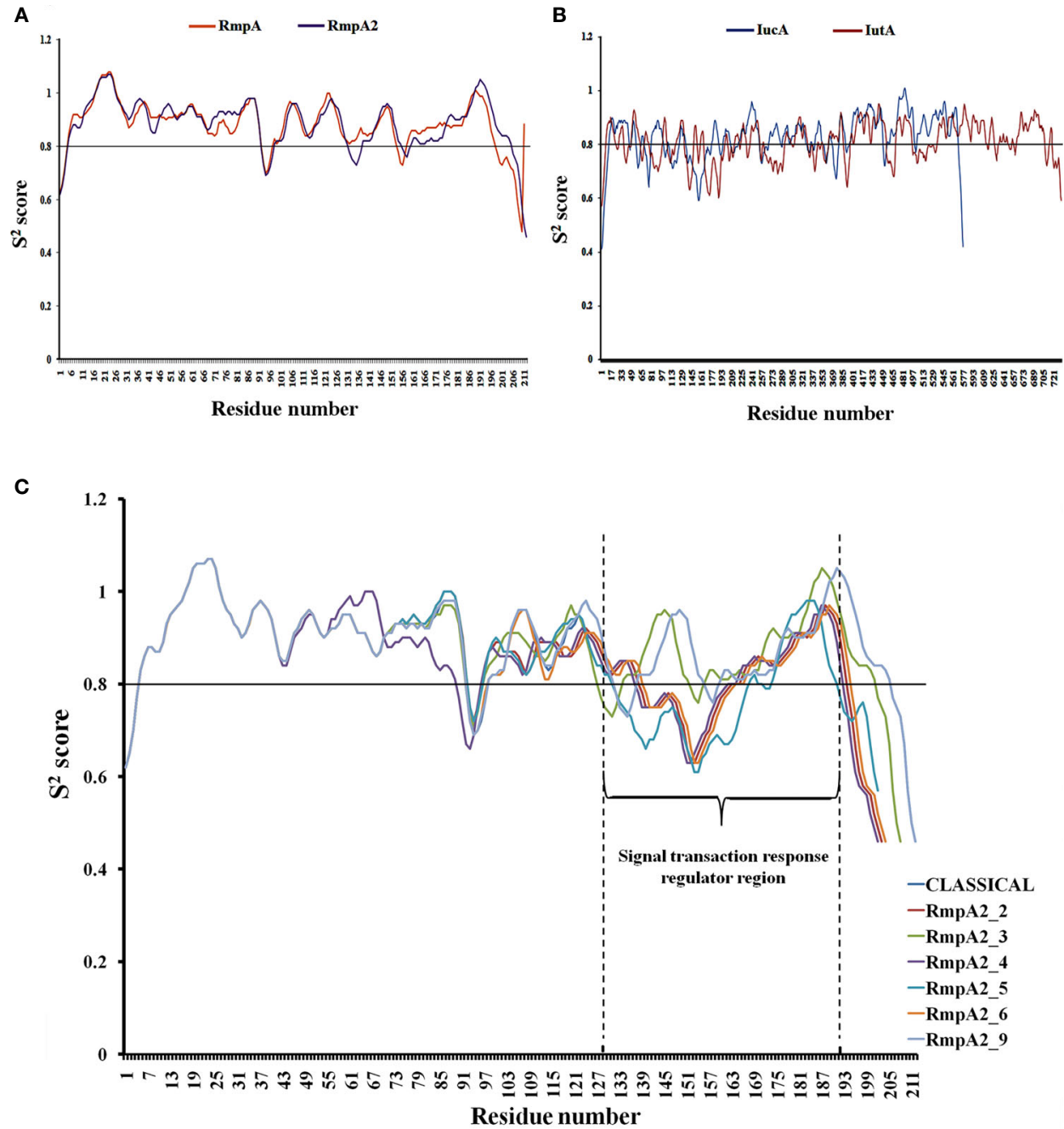

FIGURE 5 | Backbone stability analysis: The relatively rigid (S2 score >0.8) and flexible (S2 score $<0.8)$ regions of (A) RmpA and RmpA2 (B) lutA and lucA. (C) The variants of RmpA2 with shifts in the backbone as a resultant of frameshift mutations.

confirmed in animal models to establish the virulence of strains carrying aerobactin alone. In addition to virulence factors, the role of plasmids carrying antimicrobial resistance in impelling the growth rate among these isolates needs further validation.

\section{Correlation of In-Vitro and In-Silico Results}

The absence of rmpA from the virulence plasmid of most CRhvKp in the present study is warranted by the least structural stability of RmpA deduced from in silico analysis. Artemis comparison tool (Carver et al., 2005) was used to compare the plasmid compositions of the study isolate (CP067046) with reference virulence plasmid, SGH10 (CP025081) and pE16KP0290-1 (CP052259). Feature statistics showed that the relative guanine-cytosine (GC) content $(\%)$ of $r m p A(<35 \%)$ is significantly lower than that of aerobactin genes iucA and iutA (>55\%). Higher GC content not only depicts higher genomic stability but also projects an enhanced translation process and efficient amino acid usage (Li et al., 2015) of aerobactin over
rmpA. Among CR-hvKp, there is additional fitness cost when compared with CRKp or hvKp due to the presence of both resistance and virulence plasmids. Furthermore, the presence of unstable proteins imposes additional energy expenditure by the organism in maintaining the protein, which can be balanced by losing the protein. The frameshifted $r m p A 2$ with functional aerobactin is retained on the virulence plasmid taken up by the CRKp clones. As described in the previous section, aerobactin has lower fluctuations in the receptor domain with overall high backbone stability and hence has not undergone functional changes like RmpA2. Interestingly, in our experience, apart from the endemic clone ST231, other classical MDR $K$. pneumoniae that lacked a virulence plasmid and belong to diverse clones such as ST11, ST14, and ST147 did not carry aerobactin (data not shown; genomes available in PRJNA400267). Therefore, it can be inferred that the CR-hvKp has reserved aerobactin, an important virulence trait, and is prone to eliminate $r m p A$ and $r m p A 2$. 


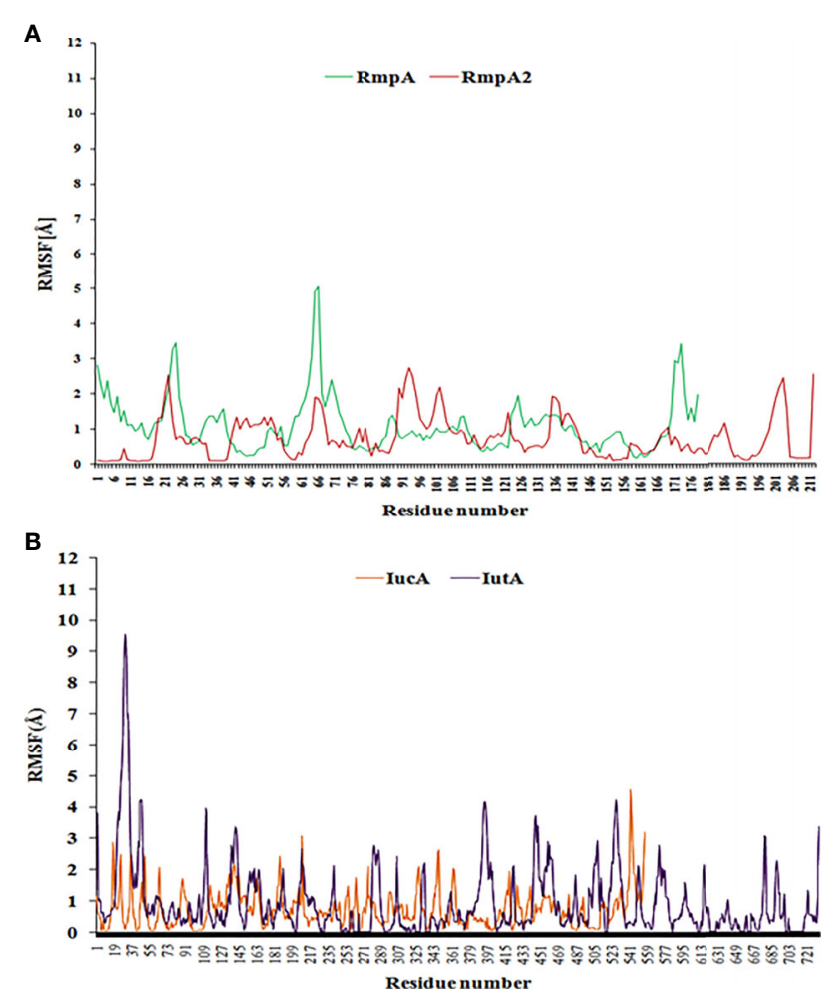

FIGURE 6 | The RMSF curve from coarse-dynamics simulations. Relative RMSF of (A) RmpA and RmpA2 (B) lucA and lutA.

\section{DISCUSSION}

Hypervirulent $\mathrm{Kp}$ is the most vicious pathotype of $\mathrm{Kp}$ that is now seen carrying antimicrobial resistance unlike its earlier counterpart (Chen et al., 2020; Zhang et al., 2020; Yang et al., 2021). Though in the last decade the virulent clones and MDR clones of Kp were distinct, the present decade has witnessed the convergence of these two groups, leading to MDR-hvKp. The formation of MDR-hvKp can be elucidated in three ways: a) acquisition of a virulence plasmid by MDR clones, b) acquisition of AMR plasmids by the virulent clones, and c) formation of mosaic plasmids that carry two replicons and code for both antimicrobial resistance and virulence (Tang et al., 2020). To the best of our knowledge, this is the first report describing the structures of RmpA, RmpA2 and variants of RmpA2 among CRhvKp belonging to high-risk international clones such as ST11, ST15, and ST231 from the Indian subcontinent. Here, the mechanism of CR-hvKp formation is due to the acquisition of a virulence plasmid by CRKp clones.

The key virulence factors among hvKp include the siderophore aerobactin, and other genes such as $r m p A, r m p A 2$, and peg-344, which are borne on the virulence plasmid. pLVPK, a $200-\mathrm{kbp}$ plasmid, a prototype of $\mathrm{Kp}$ virulence plasmid, was first identified in isolate CG43; and the loss of this plasmid conferred absence of hypermucoid colonies (Lai et al., 2003). Over the years, several screening and confirmatory methods have been used to identify hvKp, which include the string test, determining the virulence in animal/moth models and PCR detection of virulence genes (Shon et al., 2013; Russo and MacDonald, 2020). G. mellonella killing assay used in conjugation with the string test is a relatively simple and accurate method to assess $\mathrm{Kp}$ virulence and differentiate between hvKp and cKp (Russo and MacDonald, 2020). In the present study, string test followed by detection of $r m p A, \operatorname{rmp} A 2$ and aerobactin was used to define hvKp. Poor sensitivity and specificity of string test have been demonstrated earlier among ESBL-hvKp, similar to the present study results (Yu et al., 2015). The study reported absence of rmpA and mutations in $r m p A 2$ among ESBL-hvKp in addition to lack of hypermucoviscous phenotype, similar to the observations among CR-hvKp in the present study. Hence, it is evident that in the presence of AMR genes, rmpA is lost while rmpA2 is nonfunctional due to fitness cost.

The capsule polysaccharide production has three transcription units (orf1-2, orf3-15, and orf16-17) in Kp, among which two are directly influenced by $r m p A$, while one is affected by $r m p A 2$. The deletion of $r m p A$ and $r m p A 2$ leads to decreased capsular polysaccharide under anaerobic conditions (Lin et al., 2019), which is also observed in the present study. Hence, CR-hvKp that lacks $r m p A$ and $r m p A 2$ lacks hypermucoviscous phenotype due to decreased capsular polysaccharide production.

In accordance with the speculations that $\operatorname{rmpA}$ is lost among CR-hvKp, we modeled the proteins RmpA and RmpA2 to 


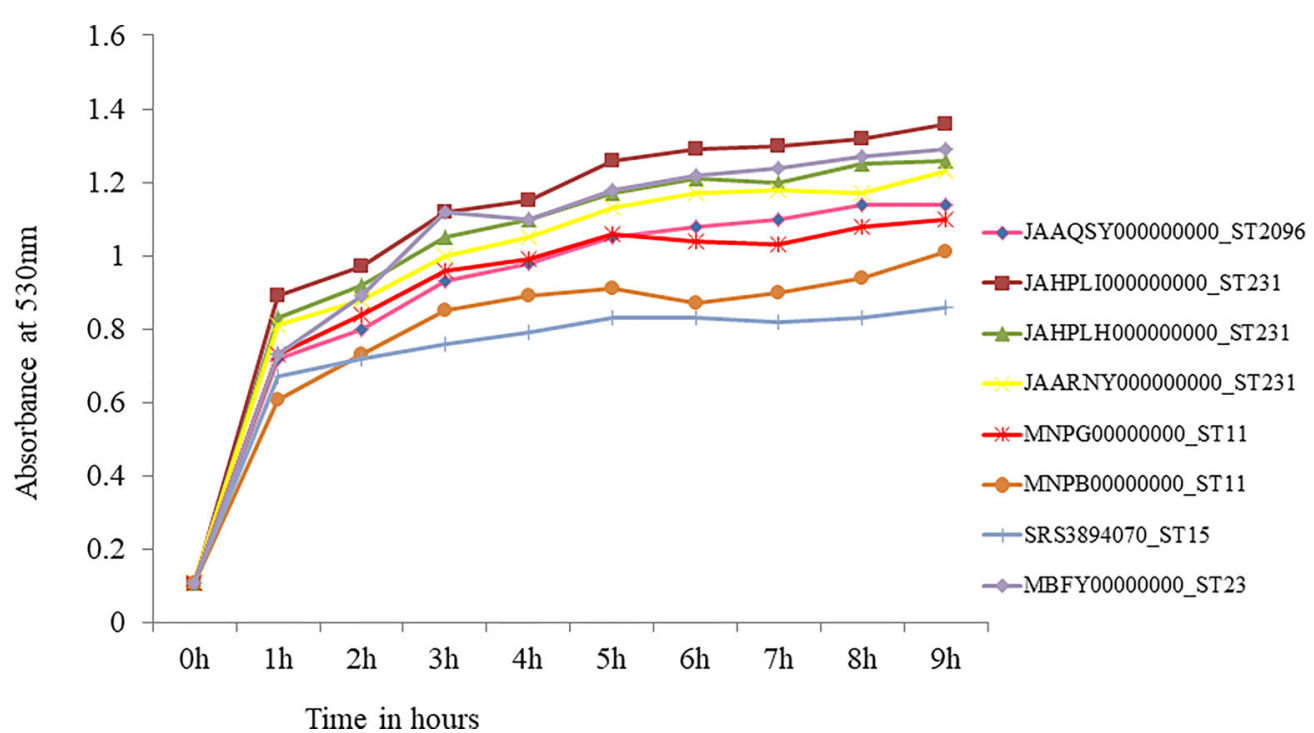

FIGURE 7 | In-vitro growth curve of hypervirulent Klebsiella pneumoniae. Isolate belonging to ST23 (MBFY00000000) was pan-susceptible and carries wild type rmpA and rmpA2. Isolates belonging to ST231 (JAHPLI000000000, JAHPLH000000000, JAARNY000000000) that carried aerobactin alone showed similar growth when compared to ST23. CRKp belonging to high risk clones ST11 (rmpA2_2, MNPG00000000; rmpA2_2, MNPB00000000) and ST15 (rmpA2_9; SRS3894070) showed slower growth than ST23 and ST231 hvKp.

determine the structural stability. It was observed that RmpA2 has a better stability profile than RmpA; and hence in MDR bacteria, RmpA2 is retained. Among the CR-hvKp, not only is the virulence plasmid losing $\operatorname{rmpA}$, but there is also the formation of a mosaic plasmid that carries $r m p A 2$ in addition to the ARGs. These events can be explained by the in silico results. It is noteworthy that the mosaic plasmids reported from our hospital earlier carried rmpA2 alone among CR-hvKp belonging to ST2096 (Shankar et al., 2020b). Hence, among CR-hvKp, string test is obsolete; and though molecular detection of $r m p A 2$ can be performed, it is essential to determine the completeness of $r m p A 2$ for gaining insights into the evolution of CR-hvKp.

In view of this, the stability profile of aerobactin-encoding proteins IucA and IutA was investigated. Aerobactin-encoding proteins had uniform high backbone rigidity and lower conformational fluctuations especially in the functional domains as compared with RmpA2 variants and hence is less prone to structural instability. Therefore, we hypothesize that CR-hvKp will retain functional aerobactin and exploit it as the key virulence factor. This is especially beneficial during the formation of mosaic plasmids that carry both virulence genes and ARGs, as it reduces the expenditure of $r m p A 2$ expression. In addition, several studies have demonstrated the prerequisite of aerobactin for survival of hvKp in animal models, and cKp lack aerobactin, while they may carry other incompetent siderophores such as enterobactin and yersiniabactin (Russo et al., 2015). Aerobactin has also been identified as an ideal anti-virulence target since it has the added advantage of being unaffected by antimicrobial resistance of the hvKp (Russo and Gulick, 2019). Table 2 lists the key virulence factors that are observed among various resistant and virulent pathotypes of $\mathrm{Kp}$ deduced from previous reports and observations. Table 3 lists the virulence factors of hvKp along with their function and location in hvKp genome.

Globally, there is a surge in the international high-risk clones acquiring virulence factors among Kp. Several studies on the acquisition of a virulence plasmid by ST11 KPC-producing Kp are increasingly reported from China, where it is endemic ( $\mathrm{Hu}$ et al., 2021). Hu and colleagues report the uptake of a virulence plasmid among $b l a_{\mathrm{KPC}-2}$ carrying ST11 Kp from various regions in China. The IncHI1B virulence plasmid in these isolates carried $r m p A$ and $r m p A 2$, or $r m p A 2$ alone. Interestingly, two clades of ST11 have been identified in China, which carries different capsule types: clade1 with K64 capsule type and clade2 with K47 capsule type (Hu et al., 2021). Cladel was associated with increased virulence factors when compared with clade2. Furthermore, $\mathrm{Li}$ and colleagues report mosaic plasmids with IncHI1B-IncFIB replicons carrying virulence gene $\operatorname{rmp} A 2$ and aerobactin among ST11 KPC Kp in China (Li et al., 2021). Notably, in the present study, CR-hvKp belonging to ST231 lacked $r m p A$ and $r m p A 2$, while it encoded aerobactin. In the present study setting, ST231 CRKp is the endemic clone predominantly carrying bla $a_{\mathrm{OXA}-232}$ on ColKP3 plasmid (Shankar et al., 2021). As speculated, for better survival of this endemic clone, aerobactin has been acquired while excluding $r m p A$ and $r m p A 2$. This is another possible evolutionary mechanism of the endemic clones acquiring a portion of the virulence plasmid that is of utmost importance while compromising on the other regions of the virulence plasmid. We hypothesize that the aerobactin might be incorporated into the chromosome of endemic clones such as ST231, which will further lead to the establishment of virulent endemic clones, causing nosocomial outbreaks. 
TABLE 2 | Key virulence markers among various pathotypes of Kp.

\begin{tabular}{|c|c|c|c|c|c|}
\hline & Classical Kp (cKp) & $\begin{array}{l}\text { Hypermucoviscous Kp } \\
\text { (hmKp) }\end{array}$ & $\begin{array}{l}\text { Hypervirulent (hvKp) } \\
\text { susceptible Kp }\end{array}$ & $\begin{array}{l}\text { Hypermucoviscous } \\
\text { hypervirulent susceptible Kp }\end{array}$ & $\begin{array}{l}\text { Hypervirulent } \\
\text { multidrug- } \\
\text { resistant Kp }\end{array}$ \\
\hline String test & - & + & $+/-$ & + & - \\
\hline $\begin{array}{l}\text { Virulence } \\
\text { plasmid }\end{array}$ & - & $+/-$ & + & + & + \\
\hline Mosaic plasmid & - & - & - & - & + \\
\hline Wild-type $r m p A$ & - & v & $+(100 \%)$ & $+(100 \%)$ & - (95\%) \\
\hline $\begin{array}{l}\text { Wild-type } \\
\text { rmpA2 }\end{array}$ & - & v & $+(100 \%)$ & $+(100 \%)$ & - \\
\hline Mutated $r m p A 2$ & - & - & - & - & $+(100 \%)$ \\
\hline Aerobactin & - & - & $+(100 \%)$ & $+(100 \%)$ & $+(100 \%)$ \\
\hline Salmochelin & - & - & $+/-(80 \%)$ & $+/-(80 \%)$ & $+/-(<10 \%)$ \\
\hline Peg-344 & - & - & $+(v)$ & $+(v)$ & $+/-(v)$ \\
\hline $\begin{array}{l}\text { Phenotypic } \\
\text { identification }\end{array}$ & Identified & Identified & Identified & Identified & unidentified \\
\hline $\begin{array}{l}\text { Genotypic } \\
\text { identification }\end{array}$ & $\begin{array}{l}\text { Not essential to designate as } \\
\qquad \mathrm{cKp}\end{array}$ & $\begin{array}{l}\text { Not essential to designate } \\
\text { as hmKp }\end{array}$ & $\begin{array}{l}\text { Mandatory to identify as } \\
\text { hvKp }\end{array}$ & Mandatory to identify as hvKp & $\begin{array}{l}\text { Mandatory to } \\
\text { identify as hvKp }\end{array}$ \\
\hline $\begin{array}{l}\text { Correlation with } \\
\text { clinical features }\end{array}$ & $\begin{array}{l}\text { Not essential to designate as } \\
\qquad \mathrm{cKp}\end{array}$ & $\begin{array}{l}\text { Not essential to designate } \\
\text { as hmKp }\end{array}$ & $\begin{array}{l}\text { Mandatory to identify as } \\
\text { hvKp }\end{array}$ & Mandatory to identify as hvKp & $\begin{array}{l}\text { Mandatory to } \\
\text { identify as hvKp }\end{array}$ \\
\hline Clones & Diverse & Diverse & CC23, CC65 & CC23, CC65 & $\begin{array}{c}\text { Regional, driven } \\
\text { by resistance } \\
\text { profile }\end{array}$ \\
\hline Clinical impact & $\begin{array}{l}\text { Less pathogenic; good clinical } \\
\text { outcome; Treatment with } \\
\text { antibiotics }\end{array}$ & $\begin{array}{c}\text { Challenges antimicrobial } \\
\text { penetration though less } \\
\text { virulent }\end{array}$ & $\begin{array}{l}\text { Fatal outcome though } \\
\text { susceptible Good biofilm } \\
\text { formers }\end{array}$ & $\begin{array}{l}\text { Fatal outcome; poor antibiotic } \\
\text { penetration Good biofilm } \\
\text { formers }\end{array}$ & Fatal outcome \\
\hline
\end{tabular}

Kp, Klebsiella pneumoniae; V, variable; CC, clonal complex.

TABLE 3 | Virulence factors among classical and hypervirulent Klebsiella pneumoniae and their function.

\begin{tabular}{|c|c|c|c|c|}
\hline Virulence factor & Function & $\begin{array}{l}\text { Location in the } \\
\text { genome }\end{array}$ & $\begin{array}{l}\text { Classical } K . \\
\text { pneumoniae }\end{array}$ & $\begin{array}{l}\text { Hypervirulent } K \text {. } \\
\text { pneumoniae }\end{array}$ \\
\hline $\begin{array}{l}\text { allABCDRS, ybbW, ybbY, fdrA, fyuA, gcl, hyi, } \\
\text { KP1_1364, KP1_1371, ylbE, ylbF }\end{array}$ & Allantoinase cluster for Allantoin metabolism & Chromosome & $x$ & $\sqrt{ }$ \\
\hline $\operatorname{arc}$ & Arginine deiminase & Chromosome & $x$ & $\sqrt{ }$ \\
\hline clbABCDEFGHIJKLMNOPQR & Colibactin gene cluster & Chromosome & $x$ & $\sqrt{ }$ \\
\hline $\operatorname{iroBCDN}$ & Salmochelin & Virulence plasmid & $x$ & $\sqrt{ }$ \\
\hline Irp1, irp2 & Yersiniabactin receptors & Chromosome & $\sqrt{ }$ & $\sqrt{ }$ \\
\hline iutA, iucABCD & Aerobactin synthesis & Virulence plasmid & $x$ & $\sqrt{ }$ \\
\hline kfuABC & Iron uptake cluster & Chromosome & $\sqrt{ } / X$ & $\sqrt{ }$ \\
\hline$g / x K, g \mid x R$ & Glycerate pathway & Chromosome & $X$ & $\sqrt{ }$ \\
\hline$k v g A, k v g S$ & $\begin{array}{l}\text { Two-component regulator system } \\
\text { regulating capsule synthesis }\end{array}$ & Chromosome & $x$ & $\sqrt{ }$ \\
\hline mceABCDEGHIJ & Microcin E492 & Chromosome & $x$ & $\sqrt{ }$ \\
\hline mrkABCDFHIJ & Type 3 fimbriae & Chromosome & $\sqrt{ }$ & $\sqrt{ }$ \\
\hline$r m p A, r m p A 2$ & Regulators of mucoid phenotype & Virulence plasmid & $x$ & $\sqrt{ }$ \\
\hline ybtAEPQSTUX & Yersiniabactin synthesis & Chromosome & $\sqrt{ }$ & $\sqrt{ }$ \\
\hline
\end{tabular}

$X$, absent; $\sqrt{ }$, present; $\sqrt{ } / X$, present/absent.

One of the most important characteristics used for identification of hvKp include the clinical presentation of the patient. In a previous study at our center, community-acquired hvKp infection showed a fatal outcome when compared with HAIs; however, the study was limited by a small sample size (Shankar et al., 2018). In the present study, a mortality of $46 \%$ can be attributed to the CR-hvKp that belong to HAI clones and hence were challenging to treat due to the limited susceptibility to antimicrobials. Interestingly, in the study cohort, there was no case of liver abscess, which explains the absence of classical hvKp presentation. It is well known that diabetes mellitus and Asian ethnicity predispose to hvKp infections (Choby et al., 2020). Mortality among hvKp-infected patients range from 30\% to $100 \%$ as reported in various studies and is also due to the antimicrobial susceptibility of the infecting hvKp (Choby et al., 2020). Unfortunately, there are no specific therapeutic choices targeting hvKp, and discrete use of antimicrobials based on susceptibility testing must be employed. In cases of localized 
infection with hvKp, source removal is the most important technique in infection management (Shon et al., 2013).

Though MDR-hvKp and CR-hvKp are being increasingly reported, there is a lack of rapid test to identify this pathotype. Future prospects include the development of immunochromatographic assays that can be deployed for rapid identification of hvKp, in which cultures of $K$. pneumoniae can be used to determine the presence of $r m p A, r m p A 2$, and aerobactin, especially among carbapenem-resistant isolates. This test can be used to target the signal transduction region in these proteins, which is disrupted in the $\operatorname{rmpA} 2$ mutant hvKp. Early identification of isolates carrying wild-type and mutated virulence genes can provide epidemiological data as well as appropriate management of the infection to reduce mortality. This test can provide an economical method for national surveillance studies, which will help to monitor the prevalence of hvKp.

The limitation of the present study includes the lack of diverse clones carrying aerobactin in the absence of $r m p A$ and $r m p A 2$. The in vitro and in silico models could not be validated using animals models to demonstrate the degree of virulence among the study isolates. Further studies involving patient outcomes and various genotypes of CR-hvKp are necessary to warrant the findings of this study. Also, of utmost importance is the genomic surveillance of CR-hvKp to determine the evolutionary changes in this pathotype.

\section{CONCLUSION}

Increasing incidence of convergence of $A M R$ and virulence is observed among Kp globally, which mainly occurs due to the uptake of a virulence plasmid by MDR clones or AMR plasmids by hvKp clones. Here, we report CR-hvKp in Indian subcontinent among MDR clones such as ST11, ST15, ST43, and ST231 that carry a virulence plasmid with non-functional RmpA2 but functional aerobactin. This highlights the significance of molecular/genomic identification of MDR-hvKp especially CR-hvKp and put in place better infection control measures to prevent the spread of this superbug. It is also imperative to determine the increasing incidence of this pathotype and monitor the clonal diversity from various geographical regions. The negative string test challenges phenotypic screening and rapid identification of CR-hvKp. Although classical RmpA2 is more stable than aerobactin, widespread mutations in $r m p A 2$ are accompanied with fitness cost to maintain non-functional RmpA2. On the other hand,

\section{REFERENCES}

Bachman, M. A., Breen, P., Deornellas, V., Mu, Q., Zhao, L., Wu, W., et al. (2015). Genome-Wide Identification of Klebsiella pneumoniae Fitness Genes During Lung Infection. MBio 6 (3), e00775-15. doi: 10.1128/mBio.00775-15

Bankevich, M. A., Breen, P., Deornellas, V., Mu, Q., Zhao, L., Wu, W., et al. (2015). Genome-Wide Identification of Klebsiella pneumoniae Fitness Genes During Lung Infection. MBio 6 (3), e00775-15. doi: 10.1128/mBio.00775-15

Basu, S., Naha, A., Veeraraghavan, B., Ramaiah, S., and Anbarasu, A. (2021). InSilico Structure Evaluation of BAG3 and Elucidating Its Association With Bacterial Infections Through Protein-Protein and Host-Pathogen Interaction Analysis. J. Cell. Biochem. doi: 10.1002/jcb.29953 high backbone stability and lower conformational fluctuations in the functional domain of aerobactin make it a better virulence factor that is retained among CR-hvKp. Therefore, aerobactin is a promising marker, as this superbug continues to evolve, reducing its fitness cost while coding for antimicrobial resistance and virulence.

\section{DATA AVAILABILITY STATEMENT}

The datasets presented in this study has been submitted to NCBI and the accession numbers are provided in Table $\mathbf{1}$.

\section{AUTHOR CONTRIBUTIONS}

CS: conceptualization, in-vitro methods-WGS, analysis, manuscript writing. SB: In silico methods and analysis, manuscript writing. BL: Manuscript correction, supervision. SS: Isolate revival, PCR. KV: Bioinformatics methods-genome assembly. PM: Isolates, supervision. SR: Validation of methods and manuscript review. BV and AA: Conceptualization, project supervision, funding acquisition, manuscript review. All authors contributed to the article and approved the submitted version.

\section{FUNDING}

This research was funded by the Indian Council of Medical Research (ICMR), Govt. of India, through the research grant IRIS-ID: 2019-0810.

\section{ACKNOWLEDGMENTS}

The authors would like to thank the staff and management bodies of the Department of Clinical Microbiology, CMCVellore, and School of Biosciences and Technology, VITVellore, for all the technical support during the research. SB sincerely thanks ICMR for his research. The authors also acknowledge Dr. Karthik Gunasekaran, Department of Medicine, Christian Medical College and Hospital, Vellore, India, for his contribution in collecting the patient details from electronic records.

Brisse, S., Fevre, C., Passet, V., Issenhuth-Jeanjean, S., Tournebize, R., Diancourt, L., et al. (2009). Virulent Clones of Klebsiella Pneumoniae: Identification and Evolutionary Scenario Based on Genomic and Phenotypic Characterization. PloS One 4 (3), 4982. doi: 10.1371/journal.pone.0004982

Carver, T. J., Rutherford, K. M., Berriman, M., Rajandream, M. A., Barrell, B. G., and Parkhill, J. (2005). ACT: The Artemis Comparison Tool. Bioinformatics 21, 3422-3423. doi: 10.1093/bioinformatics/bti553

Chen, Y. T., Chang, H. Y., Lai, Y. C., Pan, C. C., Tsai, S. F., and Peng, H. L. (2004). Sequencing and Analysis of the Large Virulence Plasmid pLVPK of Klebsiella Pneumoniae CG43. Gene 337, 189-198. doi: 10.1016/j.gene.2004.05.008

Chen, Y., Marimuthu, K., Teo, J., Venkatachalam, I., Cherng, B. P. Z., de Wang, L., et al. (2020). Acquisition of Plasmid With Carbapenem-Resistance Gene 
Blakpc2 in Hypervirulent Klebsiella Pneumoniae, Singapore. Emerg. Infect. Dis. 26, 549. doi: $10.3201 /$ eid2603.191230

Choby, J. E., Howard-Anderson, J., and Weiss, D. S. (2020). Hypervirulent Klebsiella Pneumoniae-Clinical and Molecular Perspectives. J. Inter. Med. 28 (3), 283-300. doi: 10.1111/joim.13007

Cilia, E., Pancsa, R., Tompa, P., Lenaerts, T., and Vranken, W. F. (2014). The DynaMine Webserver: Predicting Protein Dynamics From Sequence. Nucleic Acids Res. 42, 264-270. doi: 10.1093/nar/gku270

Clinical and Laboratory Standards Institute (CLSI). (2017). Performance Standards for Antimicrobial Susceptibility Testing; Twenty Seventh Informational Supplement. Wayne, PA: Clinical and Laboratory Standards Institute. CLSI document M100-S27.

Clinical and Laboratory Standards Institute (CLSI). (2018). Performance Standards for Antimicrobial Susceptibility Testing; Twenty Eighth Informational Supplement. Wayne, PA: Clinical and Laboratory Standards Institute. CLSI document M100-S28.

Clinical and Laboratory Standards Institute (CLSI). (2019). Performance Standards for Antimicrobial Susceptibility Testing; Twenty Ninth Informational Supplement. Wayne, PA: Clinical and Laboratory Standards Institute. CLSI document M100-S29.

Dong, N., Lin, D., Zhang, R., Chan, E. W. C., and Chen, S. (2018). Carriage of Bla KPC-2 by a Virulence Plasmid in Hypervirulent Klebsiella Pneumoniae. J. Antimicrob. Chemother. 73, 3317-3321. doi: 10.1093/jac/dky358

Grant, J. R., and Stothard, P. (2008). The CGView Server: A Comparative Genomics Tool for Circular Genomes. Nucleic Acids Res. 15, 181-184. doi: 10.1093/nar/gkn179

Gurevich, A., Saveliev, V., Vyahhi, N., and Tesler, G. (2013). QUAST: Quality Assessment Tool for Genome Assemblies. Bioinformatics 29, 1072-1075. doi: 10.1093/bioinformatics/btt086

Hao, Z., Duan, J., Liu, L., Shen, X., Yu, J., Guo, Y., et al. (2020). Prevalence of Community-Acquired, Hypervirulent Klebsiella Pneumoniae Isolates in Wenzhou, China. Microb. Drug Resist. 26, 21-27. doi: 10.1089/mdr.2019.0096

Heo, L., Park, H., and Seok, C. (2013). GalaxyRefine: Protein Structure Refinement Driven by Side-Chain Repacking. Nucleic Acids Res. 41, 384-388. doi: 10.1093/ nar/gkt458

Hu, D., Li, Y., Ren, P., Tian, D., Chen, W., Fu, P., et al. (2021). Molecular Epidemiology of Hypervirulent Carbapenemase-Producing Klebsiella Pneumoniae. Front. Cell. Infect. Microbiol. 11, 661218. doi: 10.3389/fcimb.2021.661218

Jacob, J. J., Anandan, S., Venkatesan, M., Neeravi, A., Vasudevan, K., Pragasam, A. K., et al. (2019). Genomic Analysis of Human Invasive Salmonella Enterica Serovar Typhimurium ST313 Isolate B3589 From India. Infect. Genet. Evol. 73, 416-424. doi: 10.1016/j.meegid.2019.05.023

Jamroz, M., Kolinski, A., and Kmiecik, S. (2013). CABS-Flex: Server for Fast Simulation of Protein Structure Fluctuations. Nucleic Acids Res. 41, 427-431. doi: $10.1093 /$ nar/gkt332

Kaplan, W., and Littlejohn, T. G. (2001). Swiss-PDB Viewer (Deep View). Brief. Bioinform. 2, 195-197. doi: 10.1093/bib/2.2.195

Koren, S., Walenz, B., Berlin, K., Miller, J., Bergman, N., and Phillippy, A. (2017). Canu: Scalable and Accurate Long-Read Assembly via Adaptive $\mathrm{K}$-Mer Weighting and Repeat Separation. Genome Res. 27, 722-736. doi: 10.1101/ 071282

Lai, Y. C., Peng, H. L., and Chang, H. Y. (2003). RmpA2, an Activator of Capsule Biosynthesis in Klebsiella Pneumoniae CG43, Regulates K2 Cps Gene Expression at the Transcriptional Level. J. Bacteriol. 185, 788-800. doi: 10.1128/JB.185.3.788-800.2003

Lam, M. M., Wick, R. R., Watts, S. C., Cerdeira, L. T., Wyres, K. L., and Holt, K. E. (2021). A genomic surveillance framework and genotyping tool for Klebsiella pneumoniae and its related species complex. Nature Commun. 12 (1), 1-16.

Lam, M. M. C., Wyres, K. L., Wick, R. R., Judd, L. M., Fostervold, A., Holt, K. E., et al. (2019). Convergence of Virulence and MDR in a Single Plasmid Vector in MDR Klebsiella Pneumoniae ST15. J. Antimicrob. Chemother. 74, 1218-1222. doi: 10.1093/jac/dkz028

Lavanya, P., Ramaiah, S., and Anbarasu, A. (2013). Influence of CHO Interactions on the Structural Stability of $\beta$-Lactamases. J. Biol. Phys. 39, 649-663. doi: $10.1007 / \mathrm{s} 10867-013-9324-\mathrm{x}$

Lavanya, P., Ramaiah, S., and Anbarasu, A. (2014). Computational Analysis of N$\mathrm{H} \cdots \pi$ Interactions and Its Impact on the Structural Stability of $\beta$-Lactamases. Comput. Biol. Med. 46, 22-28. doi: 10.1016/j.compbiomed.2013.12.008
Lavanya, P., Ramaiah, S., Singh, H., Bahadur, R., and Anbarasu, A. (2015). Investigations on the Role of $\mathrm{CH} \ldots \mathrm{O}$ Interactions and Its Impact on Stability and Specificity of Penicillin Binding Proteins. Comput. Biol. Med. 65, 85-92. doi: 10.1016/j.compbiomed.2015.07.028

Li, P., Liang, Q., Liu, W., Zheng, B., Liu, L., Wang, W., et al. (2021). Convergence of Carbapenem Resistance and Hypervirulence in a Highly-Transmissible ST11 Clone of K. Pneumoniae: An Epidemiological, Genomic and Functional Study. Virulence 12, 377-388. doi: 10.1080/21505594.2020.1867468

Lin, T. H., Wu, C. C., Kuo, J. T., Chu, H. F., Lee, D. Y., and Lin, C. T. (2019). FnrDependent Rmpa and Rmpa2 Regulation of Capsule Polysaccharide Biosynthesis in Klebsiella Pneumoniae. Front. Microbiol. 10, 2436. doi: $10.3389 /$ fmicb.2019.02436

Li, J., Zhou, J., Wu, Y., Yang, S., and Tian, D. (2015). GC-Content of Synonymous Codons Profoundly Influences Amino Acid Usage. G3 Genes. Genomes. Genet. 5, 2027-2036. doi: 10.1534/g3.115.019877

Magiorakos, A. P., Srinivasan, A., Carey, R. B., Carmeli, Y., Falagas, M. E., Giske, C. G., et al. (2012). Multidrug-Resistant, Extensively Drug-Resistant and Pandrug-Resistant Bacteria: An International Expert Proposal for Interim Standard Definitions for Acquired Resistance. Clin. Microbiol. Infect. 18, 268-281. doi: 10.1111/j.1469-0691.2011.03570.x

Parks, D. H., Imelfort, M., Skennerton, C. T., Hugenholtz, P., and Tyson, G. W. (2015). CheckM: Assessing the Quality of Microbial Genomes Recovered From Isolates, Single Cells, and Metagenomes. Genome Res. 25, 1043-1055. doi: $10.1101 /$ gr.186072.114

Pettersen, E. F., Goddard, T. D., Huang, C. C., Couch, G. S., Greenblatt, D. M., Meng, E. C., et al. (2004). UCSF Chimera-A Visualization System for Exploratory Research and Analysis. J. Comput. Chem. 25, 1605-1612. doi: $10.1002 /$ jcc. 20084

Pugalenthi, G., Shameer, K., Srinivasan, N., and Sowdhamini, R. (2006). HARMONY: A Server for the Assessment of Protein Structures. Nucleic Acids Res. 34, 231-234. doi: 10.1093/nar/gkl314

Russo, T. A., and Gulick, A. M. (2019). Aerobactin Synthesis Proteins as Antivirulence Targets in Hypervirulent Klebsiella Pneumoniae. ACS Infect. Dis. 5, 1052-1054. doi: 10.1021/acsinfecdis.9b00117

Russo, T. A., and MacDonald, U. (2020). The Galleria Mellonella Infection Model Does Not Accurately Differentiate Between Hypervirulent and Classical Klebsiella Pneumoniae. mSphere 5 (1), e00850-19. doi: 10.1128/ msphere.00850-19

Russo, T. A., MacDonald, U., Hassan, S., Camanzo, E., LeBreton, F., Corey, B., et al. (2021). An Assessment of Siderophore Production, Mucoviscosity, and Mouse Infection Models for Defining the Virulence Spectrum of Hypervirulent Klebsiella Pneumoniae. mSphere 6 (2), e00045-21. doi: 10.1128/ msphere.00045-21

Russo, T. A., Olson, R., Fang, C. T., Stoesser, N., Miller, M., MacDonald, U., et al. (2018). Identification of Biomarkers for Differentiation of Hypervirulent Klebsiella Pneumoniae From Classical K. Pneumoniae. J. Clin. Microbiol. 56 (9), e00776-18. doi: 10.1128/JCM.00776-18

Russo, T. A., Olson, R., MacDonald, U., Beanan, J., and Davidsona, B. A. (2015). Aerobactin, But Not Yersiniabactin, Salmochelin, or Enterobactin, Enables the Growth/Survival of Hypervirulent (Hypermucoviscous) Klebsiella Pneumoniae Ex Vivo and In Vivo. Infect. Immun. 83, 3325-3333. doi: 10.1128/IAI.00430-15

Shankar, C., Jacob, J. J., Sugumar, S. G., Natarajan, L., Rodrigues, C., Mathur, P., et al. (2021). Distinctive Mobile Genetic Elements Observed in the Clonal Expansion of Carbapenem-Resistant Klebsiella Pneumoniae in India. Microb. Drug Resist 27 (8), 1096-1104. doi: 10.1089/mdr.2020.0316

Shankar, C., Jacob, J. J., Vasudevan, K., Biswas, R., Manesh, A., Sethuvel, D. P. M., et al. (2020a). Emergence of Multidrug Resistant Hypervirulent ST23 Klebsiella Pneumoniae: Multidrug Resistant Plasmid Acquisition Drives Evolution. Front. Cell. Infect. Microbiol. 10, 575289. doi: 10.3389/fcimb.2020.575289

Shankar, C., Vasudevan, K., Jacob, J. J., Baker, S., Isaac, B. J., Neeravi, A. R., et al. (2020b). Mosaic Antimicrobial Resistance/Virulence Plasmid in Hypervirulent ST2096 Klebsiella Pneumoniae in India: The Rise of a New Superbug? bioRxiv. doi: 10.1101/2020.12.11.422261

Shankar, C., Veeraraghavan, B., Nabarro, L. E. B., Ravi, R., Ragupathi, N. K. D., and Rupali, P. (2018). Whole Genome Analysis of Hypervirulent Klebsiella Pneumoniae Isolates From Community and Hospital Acquired Bloodstream Infection. BMC Microbiol. 18 (1), 1-9. doi: 10.1186/s12866-017-1148-6 
Shon, A. S., Bajwa, R. P. S., and Russo, T. A. (2013). Hypervirulent (Hypermucoviscous) Klebsiella Pneumoniae: A New and Dangerous Breed. Virulence 15, 107-118. doi: 10.4161/viru.22718

Sullivan, M. J., Petty, N. K., and Beatson, S. A. (2011). Easyfig: A Genome Comparison Visualizer. Bioinformatics 27, 1009-1010. doi: 10.1093/bioinformatics/btr039

Tan, T. Y., Cheng, Y., Ong, M., and Ng, L. S. Y. (2014). Performance Characteristics and Clinical Predictive Value of the String Test for Detection of Hepato-Virulent Klebsiella Pneumoniae Isolated From Blood Cultures. Diagn. Microbiol. Infect. Dis. 78, 127-128. doi: 10.1016/j.diagmicrobio.2013.10.014

Tang, M., Kong, X., Hao, J., and Liu, J. (2020). Epidemiological Characteristics and Formation Mechanisms of Multidrug-Resistant Hypervirulent Klebsiella Pneumoniae. Front. Microbiol. 11, 581543. doi: 10.3389/fmicb.2020.581543

Tatusova, T., Dicuccio, M., Badretdin, A., Chetvernin, V., Nawrocki, E. P., Zaslavsky, L., et al. (2016). NCBI Prokaryotic Genome Annotation Pipeline. Nucleic Acids Res. 44, 6614-6624. doi: 10.1093/nar/gkw569

Turton, J., Davies, F., Turton, J., Perry, C., Payne, Z., and Pike, R. (2019). Hybrid Resistance and Virulence Plasmids in "High-Risk" Clones of Klebsiella Pneumoniae, Including Those Carrying Blandm-5. Microorganisms 7, 326. doi: 10.3390/microorganisms7090326

Vasudevan, K., Devanga Ragupathi, N. K., Jacob, J. J., and Veeraraghavan, B. (2020). Highly Accurate-Single Chromosomal Complete Genomes Using IonTorrent and MinION Sequencing of Clinical Pathogens. Genomics 112, 545-551. doi: 10.1016/j.ygeno.2019.04.006

Veeraraghavan, B., Shankar, C., Karunasree, S., Kumari, S., Ravi, R., and Ralph, R. (2017). Carbapenem Resistant Klebsiella Pneumoniae Isolated From Bloodstream Infection: Indian Experience. Pathog. Glob. Health 111, 240246. doi: 10.1080/20477724.2017.1340128

Walker, B. J., Abeel, T., Shea, T., Priest, M., Abouelliel, A., Sakthikumar, S., et al. (2014). Pilon: An Integrated Tool for Comprehensive Microbial Variant Detection and Genome Assembly Improvement. PloS One 9 (11), e112963. doi: 10.1371/journal.pone.0112963

Wang, S., Li, W., Liu, S., and Xu, J. (2016). RaptorX-Property: A Web Server for Protein Structure Property Prediction. Nucleic Acids Res. 44, 430-435. doi: $10.1093 /$ nar/gkw306

Webb, B., and Sali, A. (2017). "Protein Structure Modeling With MODELLER." in Functional Genomics. Methods in Molecular Biology, vol 1654. Eds. M, Kaufmann, C, Klinger and A, Savelsbergh (New York: Humana Press), pp. 39-54. doi: 10.1007/978-1-4939-7231-9_4

Wick, R. R., Judd, L. M., Gorrie, C. L., and Holt, K. E. (2017). Unicycler: Resolving Bacterial Genome Assemblies From Short and Long Sequencing Reads. PloS Comput. Biol. 13, 1005595. doi: 10.1371/journal.pcbi.1005595
Wiederstein, M., and Sippl, M. J. (2007). ProSA-Web: Interactive Web Service for the Recognition of Errors in Three-Dimensional Structures of Proteins. Nucleic Acids Res. 35, 407-410. doi: 10.1093/nar/gkm290

Yang, X., Dong, N., Chan, E. W. C., Zhang, R., and Chen, S. (2021). Carbapenem Resistance-Encoding and Virulence-Encoding Conjugative Plasmids in Klebsiella Pneumoniae. Trends Microbiol. 29, 65-83. doi: 10.1016/ j.tim.2020.04.012

Yang, J., and Zhang, Y. (2015). I-TASSER Server: New Development for Protein Structure and Function Predictions. Nucleic Acids Res. 43, 174-181. doi: 10.1093/nar/gkv342

Yu, W. L., Lee, M. F., Tang, H. J., Chang, M. C., and Chuang, Y. C. (2015). Low Prevalence of rmpA and High Tendency of rmpA Mutation Correspond to Low Virulence of Extended Spectrum $\beta$-Lactamase-Producing Klebsiella Pneumoniae Isolates. Virulence 6, 162-172. doi: 10.1080/21505594.2015. 1016703

Zhang, Y., Jin, L., Ouyang, P., Wang, Q., Wang, R., Wang, J., et al. (2020). Evolution of Hypervirulence in Carbapenem-Resistant Klebsiella Pneumoniae in China: A Multicentre, Molecular Epidemiological Analysis. J. Antimicrob. Chemother. 75, 327-336. doi: 10.1093/jac/dkz446

Zhan, L., Wang, S., Guo, Y., Jin, Y., Duan, J., Hao, Z., et al. (2017). Outbreak by Hypermucoviscous Klebsiella Pneumoniae ST11 Isolates With Carbapenem Resistance in a Tertiary Hospital in China. Front. Cell. Infect. Microbiol. 7, 182. doi: $10.3389 /$ fcimb.2017.00182

Conflict of Interest: The authors declare that the research was conducted in the absence of any commercial or financial relationships that could be construed as a potential conflict of interest.

Publisher's Note: All claims expressed in this article are solely those of the authors and do not necessarily represent those of their affiliated organizations, or those of the publisher, the editors and the reviewers. Any product that may be evaluated in this article, or claim that may be made by its manufacturer, is not guaranteed or endorsed by the publisher.

Copyright (C) 2021 Shankar, Basu, Lal, Shanmugam, Vasudevan, Mathur, Ramaiah, Anbarasu and Veeraraghavan. This is an open-access article distributed under the terms of the Creative Commons Attribution License (CC BY). The use, distribution or reproduction in other forums is permitted, provided the original author(s) and the copyright owner(s) are credited and that the original publication in this journal is cited, in accordance with accepted academic practice. No use, distribution or reproduction is permitted which does not comply with these terms. 